\title{
Dark signal correction for a lukecold frame-transfer CCD
}

\section{New method and application to the solar imager of the PICARD space mission}

\author{
J.-F. Hochedez ${ }^{1,2}$, C. Timmermans ${ }^{3}$, A. Hauchecorne ${ }^{1}$, and M. Meftah ${ }^{1}$ \\ 1 Laboratoire Atmosphères, Milieux, Observations Spatiales (LATMOS), Centre National de la Recherche Scientifique (CNRS), \\ Université Paris VI, Université de Versailles Saint-Quentin-en-Yvelines, Institut Pierre-Simon Laplace (IPSL), 78280 Guyancourt, \\ France \\ e-mail: hochedez@latmos.ipsl.fr \\ 2 Observatoire Royal de Belgique - Koninklijke Sterrenwacht van België (ORB-KSB), Allée Circulaire 3, 1180 Bruxelles (Uccle), \\ Belgium \\ e-mail: hochedez@oma.be \\ 3 Institut de Statistique, Biostatistique et Sciences Actuarielles (ISBA), Voie du Roman Pays 20, L1.04.01, 1348 Louvain-la-Neuve, \\ Belgium
}

Received 30 March 2013 / Accepted 28 October 2013

\begin{abstract}
Context. Astrophysical observations must be corrected for their imperfections of instrumental origin. When charge-coupled devices (CCDs) are used, their dark signal is one such hindrance. In their pristine state, most CCD pixels are cool, that is, they exhibit a low quasi-uniform dark current, which can be estimated and corrected for. In space, after having been hit by an energetic particle, pixels can turn hot, viz. they start delivering excessive, less predictable, dark current. The hot pixels therefore need to be flagged so that a subsequent analysis may ignore them.

Aims. The image data of the PICARD-SODISM solar telescope require dark signal correction and hot pixel identification. Its E2V 42$80 \mathrm{CCD}$ operates at $-7.2^{\circ} \mathrm{C}$ and has a frame-transfer architecture. Both image and memory zones thus accumulate dark current during integration and readout time, respectively. These two components must be separated in order to estimate the dark signal for any given observation. This is the main purpose of the dark signal model presented in this paper.

Methods. The dark signal time-series of every pixel was processed by the unbalanced Haar technique to timestamp when its dark signal changed significantly. In-between these instants, the two components were assumed to be constant, and a robust linear regression, with respect to integration time, provides first estimates and a quality coefficient. The latter serves to assign definitive estimates for this pixel and that period.

Results. Our model is part of the SODISM Level 1 data production scheme. To confirm its reliability, we verified on dark frames that it leaves a negligible residual bias $\left(5 \mathrm{e}^{-}\right)$and generates a small $\mathrm{rms}$ error $\left(25 \mathrm{e}^{-} \mathrm{rms}\right)$. We also examined the distribution of the image zone dark current. The cool pixel level is found to be $4.0 \mathrm{e}^{-} \mathrm{pxl}^{-1} \mathrm{~s}^{-1}$, in agreement with the predicted value. The emergence rate of hot pixels was investigated as well. It yields a threshold criterion at $50 \mathrm{e}^{-} \mathrm{pxl}^{-1} \mathrm{~s}^{-1}$. The growth rate is found to be on average $\sim 500$ new hot pixels per day, that is, $4.2 \%$ of the image zone area per year.

Conclusions. A new method for dark signal correction of a frame-transfer CCD operating near $-10{ }^{\circ} \mathrm{C}$ is described and applied. It allows making recommendations about the implementation and scientific usage of such CCDs. Moreover, aspects of the method (adaptation of the unbalanced Haar technique, dedicated robust linear regression) have a generic interest.
\end{abstract}

Key words. instrumentation: detectors - methods: data analysis - techniques: image processing

\section{Introduction}

When recorded for an intended astrophysical investigation or for any other scientific exploitation, raw observational data need to be corrected for various undesirable effects of instrumental nature. In doing so, the general goal is to reach the best possible accuracy and precision in view of a faithful estimation of the observed physical quantity. We propose a method for mitigating several interrelated instrumental effects - the dark signal and hot pixels, its outliers - which affect every scientific use of chargecoupled devices (CCDs) unless they are cooled deeply.

In the title, the CCD temperature is jestingly referred to as being "lukecold", that is, not as warm as lukewarm, but not really cold either. In the situation we are dealing with, as possibly in several others, the $\mathrm{CCD}$ is cooled down to about $-10^{\circ} \mathrm{C}$. As we describe below, this is sufficient to allow muting the dark signal generated in the image zone (IZ) during exposures that last a few seconds, but it is not cold enough to silence the hot pixels or the dark signal contributed in the memory zone (MZ) by a relatively slow readout.

The dark signal is an undesired component of the information that is recorded at each CCD picture element (pixel). It occurs because of thermally generated electric currents in the detector and is, at first order, independent of the optical quantity of interest.

A particular motivation to improve the removal of the dark signal in CCD observational data comes from the fact that it is one of the first applied instrumental corrections. Indeed, the latter are not mutually commutative, and they must be first estimated and then applied, in a certain logical order. Normally, the restoration steps proceed by rewinding the flow of information upstream so that the remediation of detector effects occurs before optical problems are addressed. As a consequence 
of the early application of the dark signal correction in the sequential cleaning of the signal, a bias at this preliminary stage propagates to posterior stages of the correction process. An under-estimation of the dark signal could convert into an overestimation of the scattered light, for example.

The study we report here is part of a larger calibration effort that aims at enabling the scientific exploitation of the PICARDSODISM solar images (Meftah et al. 2013). This motivated the development of the method, which is thus illustrated by its application to SODISM observations that are determined in particular by the frame-transfer structure of the considered CCD.

\subsection{Charge-coupled devices}

With the ever advancing CCD technology and despite the recent competition by the complementary metal-oxide-semiconductor (CMOS) active pixel sensor (APS) imaging devices, CCDs have constantly been, and still remain, detectors of choice for scientific applications since they were invented in 1969 at the Bell Telephone Laboratories (Janesick et al. 1987a; Smith 2010, and references therein).

However, their robust basic concept has remained the same: photocarriers - plus some undesired charges generated by spurious sources - are collected in a potential well created by purposely biased electrodes and/or by ion implantation that generate a fixed spatially-periodic pattern of pixels in the semiconductor crystal. This is the integration phase. At the end of it, the electric potentials of the electrodes are clocked, that is, varied temporally, such that the pixel pattern slides, forcing the stored electrical charges to transfer along the columns, and then along a perpendicular linear serial register toward an output port where the signal is converted into a voltage and somehow recorded. This is the readout phase.

For full-frame CCDs, there is no separate memory zone, and during readout, the signal remains in the frame where it has been generated during the preceding integration. This single full frame thus acts as both an image zone during the integration phase and as a memory zone during the readout phase. Consequently, the operations of full-frame CCDs necessitate the implementation of a mechanical shutter to create the darkness that is required during readout. They also prevent the possibility of starting a new integration before the end of the readout of the previous frame. Frame-transfer CCDs offer an architecture that remedies these two problems. For them, the IZ frame and the (blind) MZ frame are indeed distinct, though adjacent. Inserting a rapid frame transfer in between integration and readout allows (1) avoiding the need for an external shutter; and (2) integrating afresh in the IZ while reading out the MZ in parallel. This scheme works best when the integration and readout phases have the same duration. See Fig. 3 for a schematic representation of the frame-transfer CCD concept.

The unavoidable imperfections of the above physical process and the corrections that are required to mitigate the resulting limitations have been studied by all manufacturers and scientific users of CCDs (e.g., Rodricks \& Venkataraman 2005; Burke et al. 2005), and especially at the occasion of every space instrument embarking one (e.g., Defise et al. 1997; Lo \& Srour 2003; Sirianni et al. 2004; Schou 2004; Penquer et al. 2009; Gilard et al. 2010).

When CCDs are included in an instrumental setup such as a telescope, the first correction consists in subtracting the readout electronic offset or bias. This first step involves the sampling of non-physical underscan pixels. In Appendix B.5, we discuss this offset correction in the context of our particular application.
The assessment of the dark signal is the next necessary calibration step, and it can be a delicate one, as we will see. There are still several other performance problems that stem from the sensing of light and its conversion in a digital image by a CCD camera. The list of CCD problematics includes the calibration of the quantum efficiency (QE), the knowledge of the video gain, the monitoring of non ideal flatfields, the subtraction of image residuals (Rest et al. 2002; Crisp 2011) and of cosmic-ray hits (CRHs; Hill et al. 1997; Ipatov et al. 2007), the taking into consideration of the worsening of the charge transfer inefficiency (CTI; Rhodes et al. 2010; Baggett et al. 2012), etc.

\subsection{Dark current}

We here address the dark signal in frame-transfer CCDs and the dark noise that is associated to it. Note that the dark signal is an (unsolicited) signal, and the dark noise is the (undesired) dark signal variability, measured for instance by its RMS deviation (RMSD). Furthermore, these two distinct notions must not be confused either with a third one, the read noise $(\mathrm{RN})$, which originates in the readout port of the CCD and in the amplification stages of the analog video chain of the camera electronics.

The dark signal found in a CCD pixel comes from the socalled dark current that is accumulated during the electronic integration and readout times. The dark current is generated by the thermal excitation of valence electrons into the conduction band and by the simultaneous or subsequent collection of these electrons into the potential well of the pixel. It is said to be dark because it occurs even when no light arrives at the CCD, for example, when the shutter mechanism is closed.

The dark signal is commonly believed to grow linearly with the CCD integration time and to simply add arithmetically to the other sources of signal, namely the photoelectrons induced by the impinging light and the electrons produced by CRHs. Although some departure from linearity has been shown and modeled (Widenhorn et al. 2008; Widenhorn et al. 2010; Dunlap et al. 2011, 2012), the previous linearity statements remain preponderantly valid. It will be assumed and exploited in our proposed method.

There are mainly three sources of dark current: the surface, the depletion and the diffusion dark current (Widenhorn et al. 2002).

The surface dark current arises at the interface between the silicon and the oxide, below the electrodes. To restrain it, the multi-pinned phase (MPP) structure and operations are usually implemented. For a buried n-type channel CCD, this consists in negatively biasing the electrode phases and adding a pinning implant under some of them. This enables signal integration with the $\mathrm{Si} / \mathrm{SiO}_{2}$ interface fully inverted. The holes accumulated at the interface tend to curtail the associated surface dark current by several orders of magnitude. But some surface dark current can still contribute to the signal, especially when the charges are transfered, that is, during the frame transfer stage, if there is one, and during the readout stage.

The depletion - or bulk - dark current is produced directly in the depletion zone, where the electrical field of the corresponding electrode determines the potential well of the pixel.

The diffusion dark current is generated in the field-free region below the depletion zone. In buried-channel CCDs, only the two latter components contribute significantly, but with different proportions, to the total dark signal. 


\subsection{Hot pixels}

The dark current is not uniform. Quite the reverse, CCDs exhibit a number of so-called hot pixels that deliver spikes of dark current that can be orders of magnitude stronger than elsewhere in the frame. Spatially, they are randomly distributed and appear as white dots in dark images. They create an extended tail in the dark current distribution, and this tail chiefly determines the dark signal non uniformity (DSNU; e.g., Gilard et al. 2008, 2010).

A hot pixel is caused by a local discrepancy with regard to the perfect semiconducting crystal. This can be impurities or other crystallographic defects incorporated at the manufacturing stage. However, the advent of hot pixels in flight must be attributed to damages caused by protons, neutrons, electrons, alpha particles, heavy ions, pions, gammas, or other energetic particles. These can be of cosmic or solar origin, and many are trapped in the Van Allen belts (Feynman \& Gabriel 2000). PICARD crosses the inner belt at the South Atlantic Anomaly (SAA) several times per day. This is probably the main driver for the ignition of hot pixels in its CCD.

A single event has indeed enough energy to produce either a transient ionizing effect that appears in a single frame and is loosely labeled as a CRH, whatever its origin, or permanent damage that makes the pixel hot from then on. In the case of inverted mode operations granted by an MPP architecture, the damages are believed to be mostly displacements, typically induced by non ionizing inelastic proton collisions (Srour et al. 2003; Srour \& Palko 2006; Penquer et al. 2009). This is why the mechanisms by which energetic protons degrade the charge transfer efficiency $(C T E=1-C T I)$ and produce hot pixels, have been studied thoroughly for many years (Hopkinson et al. 1996; Gilard et al. 2008, for example). The interaction with neutrons has received attention as well (Chugg et al. 2003).

Different studies have characterized the statistical behavior of hot pixels, showing in particular that they demonstrate the phenomenology of random telegraph signals (RTS; Hopkins \& Hopkinson 1993; Chugg et al. 2003; Hopkinson et al. 2007).

The capacity to anneal them by baking out the CCD, viz. heating it to temperatures higher than the cold operating conditions, has also been investigated (e.g., Defise et al. 1997; Sirianni et al. 2004; Polidan et al. 2004; Baggett et al. 2012). In parallel, p-type channel CCDs are developed with some preliminary success in an attempt to surpass the optimized radhardness of n-type channel CCDs (Marshall et al. 2004; Gow et al. 2012). Note that, albeit a nuisance, the hot pixels can serve as a diagnostic tool to correct for the CTI (Massey et al. 2010).

\subsection{Technical and data processing solutions for limiting the effects of the dark current}

For a given CCD, after design solutions have been implemented (e.g., Bogaart et al. 2009) and manufacturing care observed, the main and only true solution to limit the harmful effects of the dark signal is to cool the device. The dark current is indeed strongly (quasi exponentially) dependent on the temperature of the silicon crystal (see Widenhorn et al. 2002, and Eqs. (1) and (2) below). But even cooled, there is still always a residual dark signal contribution, particularly because hot pixels are the result of impurities or crystalline defects that reveal themselves in the bulk component of the dark current, which prevails at low temperatures. Additionally, cooling the CCD sufficiently and regulating its temperature precisely requires considerable resources that are not readily available to all space instruments. Consequently, the CCD device cannot always be cooled as much or regulated as well as it should, even in major projects (Brown \& Davies 2003). In this context, regular bakeouts (periods of time during which the CCD is heated, which allows annealing the hot pixels) offer complementary benefits to the cold conditions of scientific acquisitions.

Within the range of temperatures where the CCD is normally operated, the data must be corrected for the dark signal if its amplitude perturbs the measurement and if the dark signal correction is indeed susceptible to enable the intended scientific exploitation or to condition the other required instrumental corrections. To this aim, the typical (as well as minimal) approach is to record a dark frame just before or just after the exposed image, both taken with the same integration time, and to subtract the former from the latter. This method is straightforward but the mentioned subtraction quadratically increases the noise in the image of interest by the Poisson noise of the used instantaneous dark frame. It therefore impacts negatively the signal-to-noise ratio $(\mathrm{S} / \mathrm{N})$. To allay this, a master dark frame is sometimes generated from multiple dark frames. However, the $\mathrm{S} / \mathrm{N}$ improvement offered by this master dark frame is then hampered by a lower duty cycle for the camera. It is often impossible to record a different dark frame for all different integration times that are used by illuminated images. And it is then even more difficult to acquire the many dark frames needed to compute several master dark frames.

Various dedicated solutions have been proposed by different authors (Hill et al. 1997; Widenhorn et al. 2007; Gilard et al. 2010; Cai et al. 2010, for example). Although this is not the purpose of the present work, we additionally mention the approach of Gomez Rodriguez et al. (2009) and Burger et al. (2011), who intended to not only correct for the dark signal but also to minimize the associated dark noise.

The subject of the present paper is to report about a method that has been developed to estimate and update the dark signal of the frame-transfer CCD onboard the PICARD-SODISM solar telescope from the space data themselves. Section 2 presents the relevant specifics of the considered payload and of its space operations. Section 3 presents the principle and the details of the reconstruction method that leads to the desired dark signal model (DSM). Section 4 analyses and discusses the products of our DSM. The last section summarizes the results and concludes.

\section{Description of the context}

\subsection{PICARD mission and the SODISM instrument}

The imaging device of which the dark signal is modeled here is the flight CCD of the SODISM experiment onboard the PICARD space mission. PICARD was successfully launched on 15 June 2010 into a Sun-synchronous dawn-dusk orbit, and commissioned in flight on 12-13 October 2010. It represents a European asset aiming at collecting solar observations that can serve to estimate some of the inputs to Earth climate models (Thuillier et al. 2006). The mission scientific payload consists of the SODISM imager and two radiometers, SOVAP (SOlar VAriability PICARD) and PREMOS (PREcision MOnitor Sensor), which carry out measurements that allow estimating the total solar irradiance (TSI) and the spectral solar irradiance (SSI) from the mid-ultraviolet to the red.

The Solar Diameter Imager and Surface Mapper (SODISM; Meftah et al. 2013) continuously acquires wide-field images of the photosphere and chromosphere of the Sun in five narrow pass bands centered at 215.0, 393.37, 535.7, 607.1 , and $782.2 \mathrm{~nm}$. It contributes images that can also feed 
SSI reconstruction models. Furthermore, the scientific objectives of SODISM encompass the probing of the interior of the Sun via helioseismic analysis of observations in intensity on the solar disk and at the limb (Corbard et al. 2008, 2013), and via astrometric investigations at the limb. The latter addresses especially the spectral dependence of the radial limb shape, and the solar diameter and solar asphericity evolution.

Given the high metrological ambitions of the original SODISM objectives, it has been recognized that its data needed to be properly corrected for all relevant instrumental problems. These corrections are indeed required to safeguard the morphometric and photometric potential of the data. Until an optical aberration became manifest, the effects of the dark signal were expected to count among the factors that would most limit several of the scientific objectives. This judgment triggered the present study.

The dark signal correction is specifically intended to support the scientific investigations by (a) rejecting the hot pixels that disrupt the morphology of solar features, including sunspots and the radial profile of the solar limb; (b) attenuating the pattern that stems from the residual DSNU, and particularly the striation ensuing from the memory zone component of the DSNU; (c) unbiasing the subsequent estimation of the other sources of spurious signal, such as scattered light and undesired reflections (ghosts), to minimize the photometric bias that may hamper scientific investigations. This last objective is quite critical because the mentioned optical effects must be estimated outside the solar disk where the various signal contaminations are all unknown, weak, and of comparable amplitudes.

In a prior stage, the SODISM telemetry is processed, together with ancillary information, to produce Level 0 (L0, or N0 for Niveau 0 in French) FITS files, including raw image data and header information. The next stage addresses the instrumental aspects to generate corrected Level 1 (L1 or N1) products and/or L1 correcting procedures, fed by auxiliary L1 data. In this context, our work represents the first step of the general L1 endeavor, and our DSM is meant to supply such auxiliary L1 data.

\subsection{SODISM CCD and associated camera electronics}

\subsubsection{Frame-transfer architecture and format of the CCD}

The SODISM Flight CCD (CCD\#60) is a device from the CCD 42-80 series of E2V. It was purchased as part of a procurement for the COnvection ROtation and planetary Transits (CoRoT) astrometric space mission, see Appendix A. It has a frame-transfer architecture, contrarily to the regular commercial devices of the CCD 42-80 series. Indeed, the CCD 42 series has the flexibility for full-frame or frame-transfer variants. The commercial devices are full-frame variants only. The reason that the full-frame version is normally made instead of the frame-transfer version (which could of course be operated in full-frame mode) is that the additional three bus lines required up the sides of the image area to drive the image and store separately make the die size slightly larger and reduce the buttability of the array for mosaic use. However, E2V has made the frame-transfer variant for several special projects, CoRoT/PICARD included. The frame-transfer architecture would allow CCD operations with no - or opened - shutter mechanism, and/or exposing the image zone while reading out the memory zone. Unfortunately, neither of these two features are used in SODISM. But the frametransfer structure does make the dark signal problem worse than it would be for a full-frame CCD, as we will show in Sect. 3. Programmatic synergies and imperfect anticipation of the problem seem to have prevailed in the decision to use a CoRoT CCD in SODISM.

The pixels of the CCD 42-80 series are square, have a size of $13.5 \times 13.5 \mu \mathrm{m}^{2}$ and no anti-blooming structures. The image zone is $2048 \times 2048$. The IZ is where the photons of interest impinge the sensor during exposure time, when the shutter is open, and where the signal accumulates during the electronic integration (see Fig. 3). The memory zone is $2048 \times 2052$. This is where the signal is stored during the readout phase. It has four lines more than the IZ because the serial register mask has four lines included with it. The MZ is masked with an optical aluminum shield that creates constant dark conditions there.

The E2V 42-80 CCDs have a buried channel structure and function in the advanced inverted mode operation (AIMO). AIMO is an E2V technology that combines the benefit of the MPP structure and operations - that is, negligible surface dark current - while limiting the ordinary reduction in full-well capacity that otherwise results from the MPP mode.

The nominal SODISM cadence is one image per minute. This is also the highest image cadence. These $60 \mathrm{~s}$ include sequentially (a) channel selection via positioning of two filter wheels (except for dark frames); (b) CCD integration in the IZ, and associated shutter operations in case of solar exposure; (c) IZ $\rightarrow \mathrm{MZ}$ frame transfer; (d) $\mathrm{CCD}$ readout of the $\mathrm{MZ}$; and (e) onboard processing, including image-formatting and compression.

\subsubsection{A priori estimation of the dark current at the operating temperature}

The SODISM CCD is cooled so as to reach acceptable dark performance. For this, a radiator that is external to the structure of SODISM evacuates the heat from the CCD via a thermal link. To make the dark signal deterministic and to precisely maintain the pixel size in both the short and the long terms, the $\mathrm{CCD}$ is heated and regulated at $-7.2 \pm 0.1{ }^{\circ} \mathrm{C}$ peak to peak (see Meftah et al. 2013, and Fig. 1). This is not very cold, but the orbit, attitude, and resources of the platform cannot offer more. At the CCD, a thermal regulation wipes out orbital and seasonal variations of the radiator. Without regulation, the CCD would reach an unsteady temperature range, wandering around $-15^{\circ} \mathrm{C}$. Temperature sensors are located below the Invar block that supports the CCD. They provide temperature measurements with a precision of $2 \mathrm{mK}$ and an accuracy of a few $\mathrm{mK}$.

According to the manufacturer's data sheet, the dark current depends on temperature following Arrhenius law,

$\frac{D C(T)}{D C_{0}(293 \mathrm{~K})}=122 T^{3} \exp \left(-\frac{6400}{T}\right) \approx 122 T^{3} \exp \left(\frac{-E_{\mathrm{g}}}{2 k_{\mathrm{B}} T}\right)$,

where $D C$ is the dark current at temperature $T[\mathrm{~K}]$ and $D C_{0}$ is the dark current at $293 \mathrm{~K}$, both expressed (for example) in $\mathrm{e}^{-} \mathrm{pxl}^{-1} \mathrm{~s}^{-1} . E_{\mathrm{g}}$ is the bandgap energy of $\mathrm{Si}$ and amounts to $\sim 1.1 \mathrm{eV} ; k_{\mathrm{B}}$ is the Boltzmann constant $\left(\sim 8.62 \times 10^{-5} \mathrm{eV} \mathrm{K}^{-1}\right)$. Note that Eq. (1) disagrees with Eq. (22) of Widenhorn et al. (2002), reproduced here,

$D C(T)=D C_{\text {diff }} T^{3} \exp \left(\frac{-E_{\mathrm{g}}}{k_{\mathrm{B}} T}\right)+D C_{\mathrm{depl}} T^{3 / 2} \exp \left(\frac{-E_{\mathrm{g}}}{2 k_{\mathrm{B}} T}\right)$,

where $D C_{\text {diff }}$ and $D C_{\text {depl }}$ are the diffusion and depletion dark currents, respectively.

Equation (1) is not justified in the data sheet. We therefore assume that E2V derived it from empirically fitting their data. 


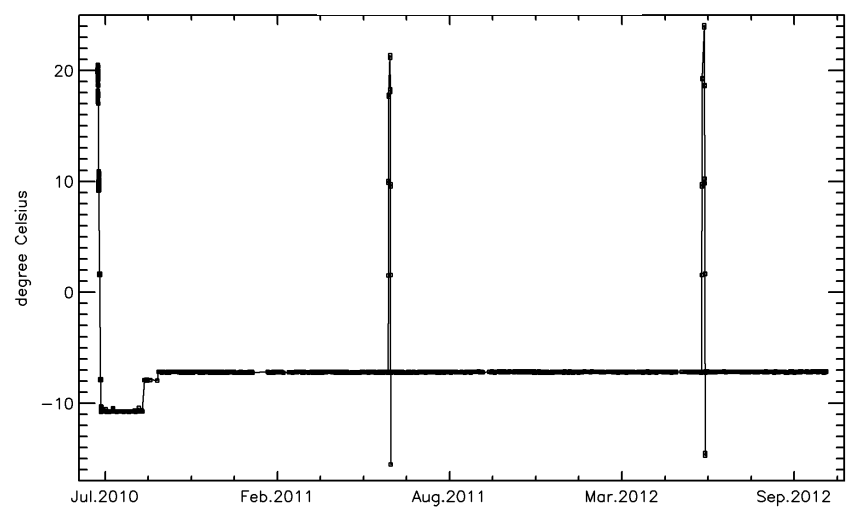

Fig. 1. Evolution of the temperature of the SODISM flight CCD. After the cooldown period in July 2010 , the CCD was first regulated at $-11^{\circ} \mathrm{C}$ for a month and a half. Because it was anticipated that this temperature could not be maintained for the whole duration of the mission, the setup point was subsequently raised to $-7.2{ }^{\circ} \mathrm{C}$ in mid September 2010. The short- and long-term stabilities around that point are on the order of $\pm 0.1{ }^{\circ} \mathrm{C}$ peak-to-peak (P-P). The precision of the measurement is $2 \mathrm{mK}$, viz. a hundredth of the amplitude of the residual variation. Two bakeout periods occurred on 15-18 June 2011 and on 13-17 June 2012. They allowed heating the CCD up to $21{ }^{\circ} \mathrm{C}$ for two days, and up to $24{ }^{\circ} \mathrm{C}$ for three days, respectively. Brief stages can be noticed during their warmup and cool-down phases.

Eq. (1) may do well at low temperatures $\left(-30^{\circ}\right.$ and below) because its activation energy employs half of the band gap, as for $D C_{\text {depl }}$, which is known to dominate there. The exponent factor on $T$ (3 or 1.5) probably does not modify the general adequateness of the fit much. However, we expect Eq. (2) to account better for the temperature dependency of the dark current because Widenhorn et al. (2002) based it on physical assumptions. Unfortunately, it would require estimating two parameters $\left(D C_{\text {diff }}\right.$ and $\left.D C_{\text {depl }}\right)$, and we have not analysed the bakeout periods (visible in Fig. 1) that would provide the needed temperature span. Our study hence cannot confirm our latter statement.

The CoRoT project had measured the typical dark current of non-hot pixels of CCD\#60, and found $0.10 \mathrm{e}^{-} \mathrm{pxl}^{-1} \mathrm{~s}^{-1}$ at $233 \mathrm{~K}=-40{ }^{\circ} \mathrm{C}$ (Lapeyrere et al. 2006, Table 1). Following Eq. (1), the SODISM flight CCD should thus exhibit a cool pixel dark current (CPDC) around $55 \mathrm{e}^{-} \mathrm{pxl}^{-1} \mathrm{~s}^{-1} \approx 4.8 \mathrm{pAcm}^{-2}$ at $293 \mathrm{~K}=+20{ }^{\circ} \mathrm{C}$ and around $4.4 \mathrm{e}^{-} \mathrm{pxl}^{-1} \mathrm{~s}^{-1}$ at $-7.2^{\circ} \mathrm{C}$. If we assume instead that the dark current is dominated by the second term of Eq. (2), non-hot pixels should exhibit a dark current on the order of $C P D C \approx 3.6 \mathrm{e}^{-} \mathrm{pxl}^{-1} \mathrm{~s}^{-1}$ at $-7.2^{\circ} \mathrm{C}$. But the diffusion component probably already increases this latter estimate by several percents.

In their CCD 42-80 datasheet, E2V also specify that the hottest pixels potentially deliver up to $4 \mathrm{e}^{-} / \mathrm{pxl} / \mathrm{h}$ at $153 \mathrm{~K}$. Following Eq. (1), they will deliver a dark current of about $4 \times 10^{6} \mathrm{e}^{-} \mathrm{pxl}^{-1} \mathrm{~s}^{-1}$ at $+20{ }^{\circ} \mathrm{C}$, and $300 \times 10^{3} \mathrm{e}^{-} \mathrm{pxl}^{-1} \mathrm{~s}^{-1}$ at $-7.2{ }^{\circ} \mathrm{C}$. If any, the hottest pixels might therefore be able to produce, during a typical one-second integration time, a dark signal that would be four times the saturation level of middle CCD pixels at their nominal operating temperature of $-7.2{ }^{\circ} \mathrm{C}$. One of our results below indicates that this does not occur. This is consistent with the datasheet since the values given by E2V are worst cases.

Appendix B provides additional information about the camera. In particular, the gain of the output chain is shown to be

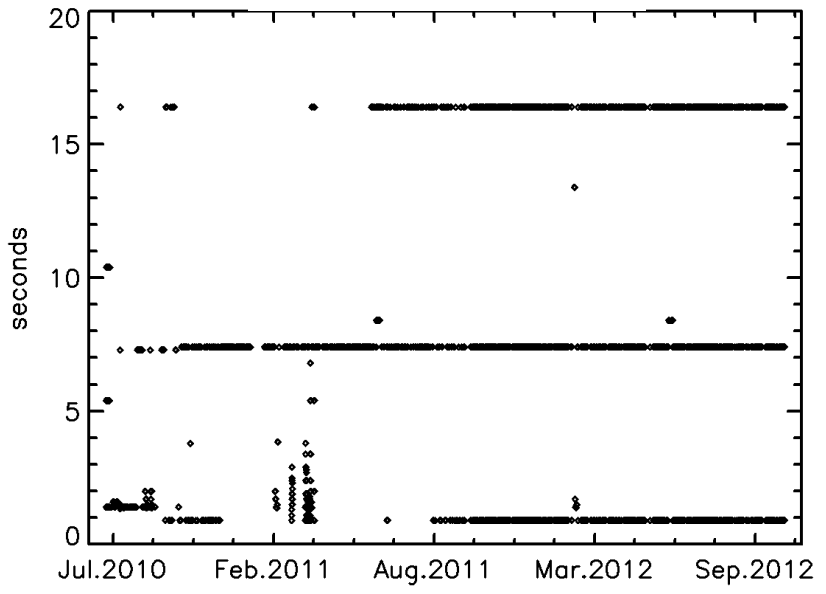

Fig. 2. Evolution of the integration time of the full CCD dark frames that are available for generating the desired dark signal model. The dark frame integration time pattern was not regular during the first year of the mission, until end of August 2011, since which time three dark frames are recorded each day with an integration time equal to $0.9,7.4$, and $16.4 \mathrm{~s}$. Before June 2011, most days and many weeks exhibit no alternation of the dark frame integration time.

$\sim 1.18 \mathrm{ADU}_{16 \mathrm{bit}} / \mathrm{e}^{-}$. We gathered in Table 1 the main parameters reported in Sect. 2.2 and in Appendix B.

\subsection{Available in-flight dark frames}

As part of the automatic ordinary science operations, also know as the SODISM routine (Meftah et al. 2013), it had been foreseen before launch to record one dark frame per day with $1.4 \mathrm{~s}$ integration time. This value is close to the nominal integration time of most channels at beginning of life (BOL). But it has been recognized during the first weeks of flight operations that dark frames with other integration times needed to be acquired. Indeed, for the $535 \mathrm{~nm}$ channel that is dedicated to helioseismology $(535 \mathrm{H})$, the exposure time is equal to $7 \mathrm{~s}$. Additionally, at the occasion of special campaigns, the exposures in any channel can be different from $1 \mathrm{~s}$. Finally, the expected degradation of the UV channels was to lead to successive increases of their exposure time, which, in 2012, has reached $16 \mathrm{~s}$ (the maximum value allowed by the camera electronics) for the $215 \mathrm{~nm}$ channel, and $6 \mathrm{~s}$ for the $393 \mathrm{~nm}$ channel.

However, PICARD is a micro-mission and some time has been required to modify the coded routine. This is why the integration time of the daily dark frames had to be alternated manually (between $0.9 \mathrm{~s}$ and $7.4 \mathrm{~s}$ ) between 25 Sep. and 2 Dec. 2010. In February and March 2011, special linearity campaigns have permitted us to vary the dark integration time at a few occasions. But for the rest, the dark exposure time was set to $7.4 \mathrm{~s}$ until 8 June 2011, when it was again possible to alternate manually between two values (7.4, and $16.4 \mathrm{~s})$ for three months, and finally from 25 August 2011 onwards, in an automatic manner among three values $(0.9,7.4$, and $16.4 \mathrm{~s})$. The dark frame program has thus been very irregular during the first year of the mission when other aspects of the performance were at their best (Fig. 2).

It should be mentioned that other dark signal images have been acquired $~ 50$ times per day from 5 Aug. 2010 until 27 Mar. 2012. However, they offer information only within a ring-shaped region spanning across the solar limb, and until now, they were not exploited by the present analysis because we are interested in 
Table 1. Parameters of the SODISM CCD camera.

\begin{tabular}{|c|c|}
\hline Parameter & Value (at $-7.2^{\circ} \mathrm{C}$ when relevant) \\
\hline CCD type & E2V CCD 42-80 series \\
\hline Flight CCD name and reference & Mehen, CCD\#60 \\
\hline Image zone format & $2048 \times 2048$ \\
\hline Memory zone format & $2048 \times 2052$ \\
\hline Pixel size & $13.5 \times 13.5 \mu \mathrm{m}^{2}$ \\
\hline$C T I$ at $\mathrm{BOL}$ & $\leq 10^{-6}$ \\
\hline Saturation level & $77-111 \mathrm{ke}^{-}$ \\
\hline Operating temperature in flight & $-7.2^{\circ} \pm 0.1 \mathrm{C}$ \\
\hline Expected dark current of non hot pixels & $\sim 4 \mathrm{e}^{-} \mathrm{pxl}^{-1} \mathrm{~s}^{-1}$ \\
\hline Expected dark current of hottest pixel & $300 \mathrm{ke}^{-} \mathrm{pxl}^{-1} \mathrm{~s}^{-1}$ \\
\hline Gain of the left readout port (image bottom) & $4.22 \mu \mathrm{V} / \mathrm{e}^{-}$ \\
\hline Gain of the right readout port (image top) & $4.14 \mu \mathrm{V} / \mathrm{e}^{-}$ \\
\hline Overall gain of the readout chain (16 bit) & $\sim 1.18 \mathrm{ADU}_{16 \mathrm{bit}} / \mathrm{e}^{-} \Rightarrow 0.85 \mathrm{e}^{-} / \mathrm{ADU}_{16 \mathrm{bit}}$ \\
\hline Overall gain of the readout chain ( 15 bit) & $\sim 0.59 \mathrm{ADU}_{15 \mathrm{bit}} / \mathrm{e}^{-} \Rightarrow 1.685 \mathrm{e}^{-} / \mathrm{ADU}_{15 \mathrm{bit}}$ \\
\hline$R N$ & Increasing from $15 \mathrm{e}^{-} \mathrm{rms}$ at $\mathrm{BOL}$, to $20 \mathrm{e}^{-} \mathrm{rms}$ at EOL \\
\hline Exposure time & $0.5-16 \mathrm{~s}$ \\
\hline Integration time & is worth "exposure time" $+0.4 \mathrm{~s}$ \\
\hline IZ-MZ frame-transfer duration & $369.46 \mathrm{~ms}$ \\
\hline Line readout duration & $11.05 \mathrm{~ms}$ \\
\hline Frame readout duration & $\sim 22 \mathrm{~s}$ \\
\hline
\end{tabular}

Notes. Abbreviations used in this table: Analog-to-digital unit (ADU), beginning of life (BOL), charge transfer inefficiency (CTI), end of life (EOL), image zone (IZ), memory zone (MZ), read noise (RN).

estimating a model of the dark signal over the whole CCD frame. In a future improvement of the present work, these other dark signal images could refine the timestamping of Step 3 described in Sect. 3.2.3 below.

\section{Modeling the dark signal from in-flight data}

After it is modeled, the dark signal correction consists simply of a subtraction since we assumed linear behavior with respect to

- its addition to the other components of the signal (desired or not), and

- integration time proportionality.

Before performing this subtraction, we needed, as a prerequisite, to model, viz. to be able to estimate, the CCD dark signal for any exposure duration and at any instant of time during the mission lifespan. This section describes a method that delivers such a DSM on the basis of in-flight dark observations scheduled with only a few different integration times that moreover were irregularly varied.

\subsection{Image zone and memory zone dark signal components}

Regarding frame-transfer CCDs, the main consideration pertains to the generation of dark current in both the image and memory zone. The dark signal is indeed the addition of an IZ component that is proportional to the programmable integration time, with an MZ component that is the sum of the contributions of all physical pixels of the MZ where the quantity of interest sojourns during readout (see Fig. 3). Practically, a pixel of coordinates $(i, j)$, having thus accumulated signal charges in row $i$ and column $j$ of the IZ, rapidly transfers its signal, just before the start of readout, to the pixel of same coordinates in the MZ. This "IZMZ" frame transfer takes only $\sim 370 \mathrm{~ms}$ and is expected to add little extra dark signal. Once in MZ, the quantity of interest is shifted $i$ times toward the serial register. During this slow process, it accumulates supplementary dark signal in each of the

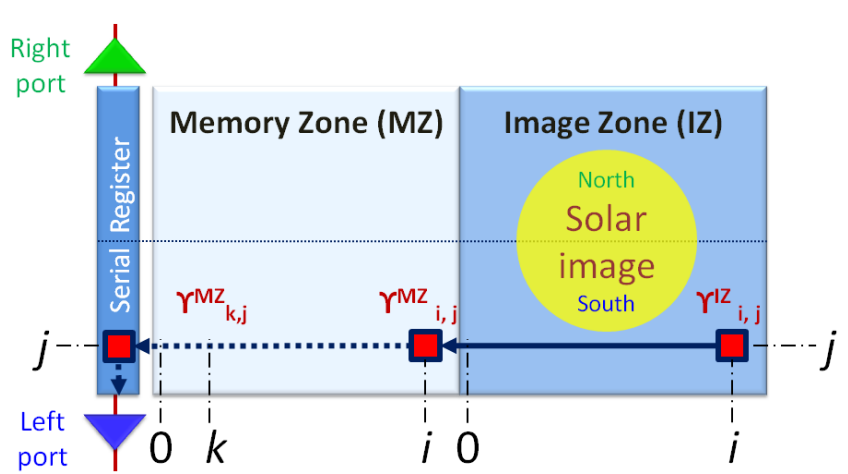

Fig. 3. Sketch of a frame-transfer CCD and scenario for the dark signal build-up. A frame-transfer CCD, such as SODISM's, has two zones, IZ and MZ, which have (quasi) the same format. During the CCD integration time, the signal is generated and stored in the IZ on the right side of the figure. This is when the shutter can be opened and the device exposed to observe the Sun. This is also when the IZ component of the dark signal accumulates at a rate of $\Upsilon_{i, j}^{\mathrm{IZ}}(t) \mathrm{e}^{-} \mathrm{pxl}^{-1} \mathrm{~s}^{-1}$. The image frame is then transferred to the MZ. At this occasion, the signal charges that were generated at coordinates $(i, j)$ of the IZ are moved to $(i, j)$ of the MZ. During the next and last phase, the readout phase, the CCD lines (represented vertically here) are transferred one by one to the serial register. The readout phase lasts $22 \mathrm{~s}$, which is sufficient to accumulate a significant amount of extra dark signal while sojourning in the $i$ pixels $(k, j)$ of the MZ.

$i$ physical pixels of the MZ, where it dwells for $11.05 \mathrm{~ms}$ while one of the foregoing CCD lines is read out.

The above scenario is formalized in Eqs. (3) and (4):

$$
\begin{aligned}
D S_{i, j}(t) & =O_{i, j}(t)+d s_{i, j}(t), \\
d s_{i, j}(t) & =G\left[(T+\delta T) \Upsilon_{i, j}^{\mathrm{IZ}}(t)+\tau \sum_{k=0}^{i} \Upsilon_{k, j}^{\mathrm{MZ}}(t)\right]
\end{aligned}
$$

where 
- $D S_{i, j}(t)$ is the random variable of the dark measurement recorded at date $t$ for the pixel of coordinates $(i, j)$. Note that the available dark images have always been recorded with 15 bit resolution.

- $O_{i, j}(t)$ is the random variable of the offset in this pixel at this time. Apart from the CCD port dependence, we consider from now on that it does not depend on the pixel because we do not have access to this information. Hence $O_{i, j}(t)=$ $O(t)$, and we used the amount that is computed onboard and provided in the L0 FITS header for the given CCD port (see Sect. B.2).

- $d s_{i, j}(t)$ is the random variable of the dark signal at $t$ for the pixel $(i, j)$.

- $G$ is the gain of the video chain (see Sect. B.2 and Eqs. (B.1) and (B.2)).

- $T$ is the programmed duration of the shutter exposure, also known as the exposure time.

$-T+\delta T=T^{\prime}$ is the duration of the CCD integration, also known as the integration time. As mentioned in Sect. B.2, $\delta T=0.4 \mathrm{~s}$.

- $\Upsilon^{\mathrm{IZ}}$ is the random variable of the dark current map in the IZ at date $t$.

- $\tau$ is the duration needed to read a CCD line. $\tau=11.05 \mathrm{~ms}$.

- $\Upsilon^{\mathrm{MZ}}$ is the random variable of the dark current map in the $\mathrm{MZ}$ at date $t$.

Equation (4) can then be rewritten as

$d s_{i, j}(t)=G \cdot\left[T^{\prime} \cdot \Upsilon_{i, j}^{\mathrm{IZ}}(t)+\tau \cdot \Psi_{i, j}^{\mathrm{MZ}}(t)\right]$,

with

$\Psi_{i, j}^{\mathrm{MZ}}(t)=\sum_{k=0}^{i} \Upsilon_{k, j}^{\mathrm{MZ}}(t)$

For every pixel, there are two unknowns, $\Upsilon^{\mathrm{IZ}}$ and $\Psi^{\mathrm{MZ}}$. In other words, we need to reconstruct the dark signal contributions of both the IZ and the MZ. This is a linear regression problem and it can be solved if there are at least two different integration times $T^{\prime}$ for a given state of $\Upsilon^{\mathrm{IZ}}$ and $\Psi^{\mathrm{MZ}}$.

Before detailing the process of reconstruction, we note in Eq. (6) that $\Psi_{i, j}^{\mathrm{MZ}}$ is a sum of positive contributions and must consequently be growing with $i$.

We also recall that a pixel is cool (viz. non-hot) until it is hit by an ionizing particle and becomes hot (see Sect. 1.3). The $d s_{i, j}(t)$ time series are thus expected to display temporally piecewise constant behaviors. They are not necessarily always growing with time because hot pixels may cool to some extent (see Fig. 5) even if they can never return completely cool.

\subsection{Generation of the dark signal model}

The goal is to estimate at any time $t, \Upsilon_{i, j}^{\mathrm{IZ}}(t)$ and $\Psi_{i, j}^{\mathrm{MZ}}(t)$, the cartographies of the IZ and MZ dark signal components. Given that Eq. (5) depends only on unknowns that are associated to a single pixel $(i, j)$, we proceeded by processing the temporal dependence CCD column per CCD column to spare computer memory, and then, pixel per pixel.

First, since integration time $T^{\prime}=7.4 \mathrm{~s}$ is the configuration that has been programmed most regularly (see Fig. 2), a provisional DSM was established for this particular $T^{\prime}$ value. The DSM/7.4s model results from the fitting of piecewise constant functions to the observed time series because the ignition and cool-down of hot pixels - whether in IZ or $\mathrm{MZ}$ - are sudden events.

Second, for every pixel, we identified the time intervals during which the dark signal of this pixel - and so, both of its IZ and $\mathrm{MZ}$ components - are constant according to DSM/7.4s.

Third, for each such interval and for each pixel, we performed a robust regression versus integration time. Next, this enabled updating the values of $\Upsilon_{i, j}^{\mathrm{IZ}}(t)$ and $\Psi_{i, j}^{\mathrm{MZ}}(t)$ for the successive periods of constancy.

Finally, the $\Upsilon_{i, j}^{\mathrm{IZ}}(t)$ and $\Psi_{i, j}^{\mathrm{MZ}}(t)$ data cubes were re-sampled on a daily grid by means of a median filter.

The next three sections (Sects. 3.2.1 to 3.2.3) describe the formation of DSM/7.4s. Its outcome is then employed in Sect. 3.2.4.

\subsubsection{Step 1: variance stabilization of the time series at $T^{\prime}=7.4 \mathrm{~s}$}

The unbalanced Haar transform (UHT) that is going to be needed in Step 3 (Sect. 3.2.3) requires the time series to be made approximately homoscedastic. This is why the $d s_{i, j}^{7.4 s}(t)$ of Eq. (3) (with $t$ chosen so that $T^{\prime}(t)=7.4 \mathrm{~s}$ ) were first of all preprocessed by a generalized Box-Cox power transform (Box \& Cox 1964; Sakia 1992):

$d s_{\mathrm{BoxCox} i, j}^{7.4 \mathrm{~s}}(t)=\frac{\left(d s_{i, j}^{7.4 s}(t)+\alpha\right)^{\lambda}-1}{\lambda \cdot \operatorname{GM}\left(d s_{i, j}^{7.4 s}\right)^{\lambda-1}}$,

where GM is the geometric mean.

Indeed, it can easily be derived from Eqs. (B.3), (B.5) and Sect. B.5 that - for the cool pixels $-d s_{\operatorname{BoxCox} i, j}^{7.4 \mathrm{~s}}(t)$ will be homoscedastic if

$\lambda=0.5$ and

$\alpha=R N^{2} \times G^{-1} \gtrsim 10[\mathrm{ADU}]^{2} \times 1.685 \approx 170 \mathrm{ADU}_{15}$ bit.

For the Jul. 2010-Nov. 2012 period, the lowest $d s_{i, j}^{7.4 s}(t)$ observation has been $-178 \mathrm{ADU}_{15}$ bit, which agrees with the $\alpha$ value that is required to variance-stabilize the quadratic addition of read noise and shot noise. The above determined Box-Cox transform will of course be less effective on hot pixels, but it a posteriori appears to be sufficient for the purpose of the UHT.

The Box-Cox preprocessed time series of CCD column \#1341 is represented in Fig. 4a.

\subsubsection{Step 2: median filtering of the time series at $T^{\prime}=7.4 \mathrm{~s}$}

Real observational data are cluttered with various outliers. In particular, telemetry missing-blocks (TMBs) and CRHs introduce spiky features in the time series of most pixels. These are fortunately easy to correct for by using a median filter computed over seven successive samples. It flags the signal when it departs from the running median by more than five running $\sigma$. When this occurs, the information is declared erroneous and replaced by the said median.

\subsubsection{Step 3: unbalanced Haar transform of the time series at $T^{\prime}=7.4 \mathrm{~s}$}

The sought DSMs, and DSM/7.4s in particular, are anticipated to be piecewise constant (viz. staircase) functions of time. Indeed, the advent of hot pixels or their partial cool-down, either in IZ or MZ, should create perfect steps in the DSM/7.4s time series. If the transition occurs in the IZ, it will be of relatively large amplitude, while the ignition of a hot pixel in the $\mathrm{MZ}$ is expected 
(a) After Step 1

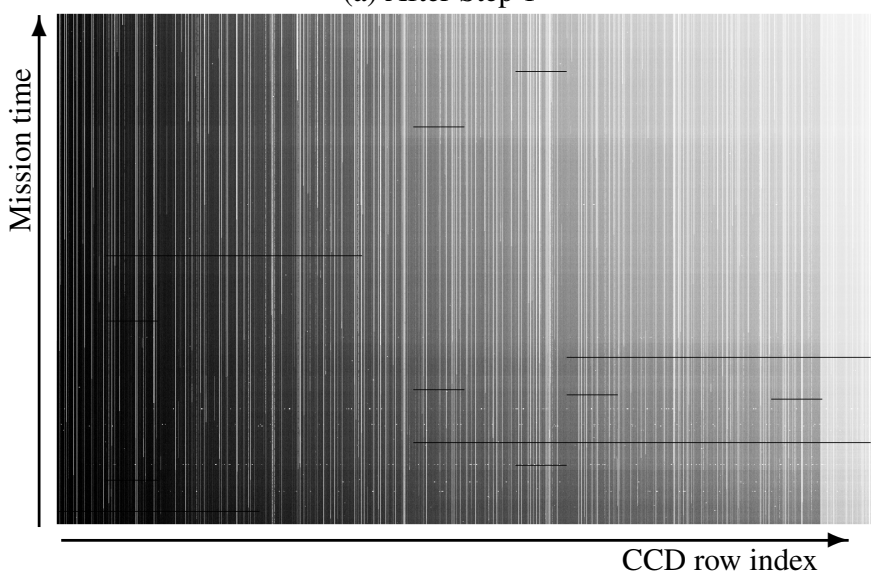

(b) After Step 3

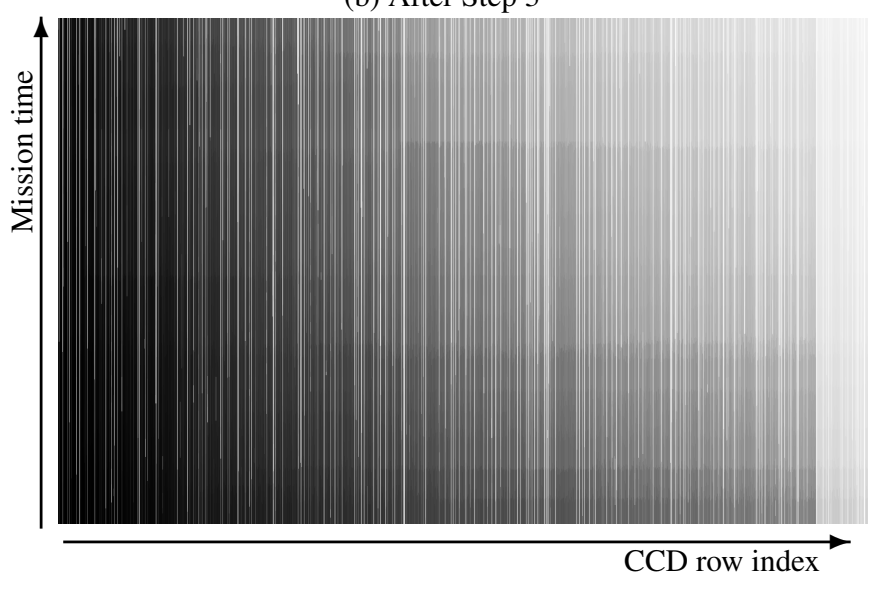

Fig. 4. a) Evolution of the dark signal in CCD column (image row) \#1341 for an integration time $T^{\prime}$ equal to $7.4 \mathrm{~s}$. The Box-Cox transformed dark signal of this CCD column are represented horizontally (after Step 1), and the information from all frames with $T^{\prime}=7.4 \mathrm{~s}$ has been stacked vertically. The presence or advent of hot pixels can be seen as bright semi-infinite vertical lines, telemetry holes as black horizontal segments, cosmic-ray hits as white dots. It can be verified that the pixels in the right part of the figure exhibit higher dark current since they dwell for a longer duration in the memory zone. We note that in this particular column \#1341, a hot pixel in the MZ generates a dark signal excess that affects the last $\sim 10 \%$ of the CCD column, on the right side of the figure. b) The same data, but after Step 3.

to generate a much smaller jump (with a ratio of $\tau / T^{\prime} \approx 0.14 \%$ ). This discrepancy will be attenuated by the Box-Cox transform of Step 1, however. It remains logical to seek an algorithm that fits staircase functions with no a priori on the instant of the steps, their amplitudes, or on their number. Conversely, no change in the dark signal should occur outside interactions with an energetic particle and annealing events.

There are several such algorithms, and we selected the multiscale unbalanced Haar transform (UHT) described by Fryzlewicz (2007) because it allows approximating any time series by a piecewise constant function in which the jumps are not necessarily positioned at dyadic locations (in contrast to other wavelet schemes), and because its computational complexity is on the order of $O(n \log n)$. Moreover, Fryzlewicz (2007) demonstrated the outstanding performance of his UHT scheme (see Fryzlewicz 2007, Fig. 1, for example).

Variance stabilization is a prerequisite to the UHT technique, which motivated Steps 1 and 2. By decomposing on a Haar-like basis the median-filtered time series yielded by Step 2, the UHT

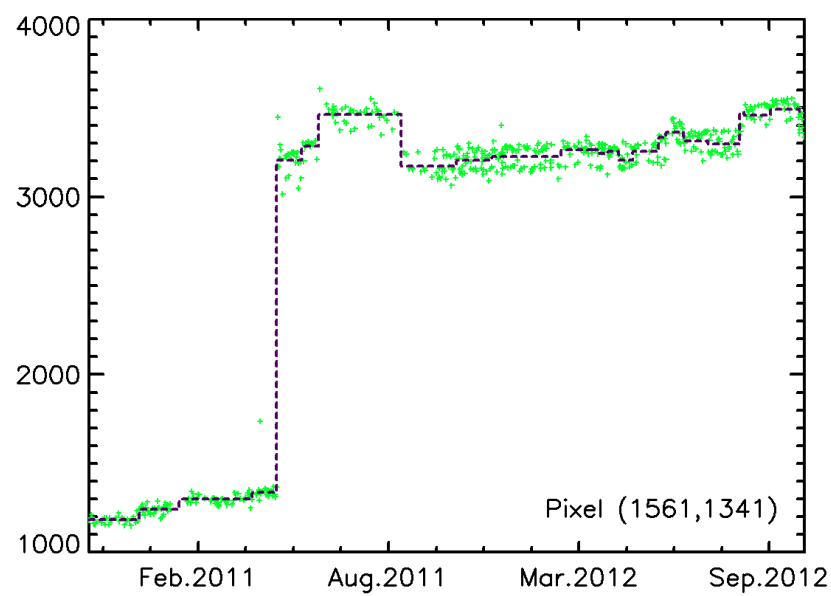

Fig. 5. Temporal evolution of the variance-stabilized dark signal $\left(T^{\prime}=\right.$ $7.4 \mathrm{~s})$ in pixel $(1561,1341)$, over-plotted with the piecewise constant fit (dashed line) that results from the reconstruction with the unbalanced Haar technique. This represents a vertical cut in Fig. 4a and the corresponding one in Fig. 4b. Note that the dark signal oscillates between two values after the pixel has become hot. This multi-level random telegraph noise is expected (cf. Sect. 1.3). The drop in dark current observed in this curve around August 2011 also illustrates and demonstrates that hot pixels cool down sometimes, even if they do not recover fully.

provides the desired instants of time in between which no hot pixel transition has taken place for the considered pixel.

Moreover, the UHT enables denoising and reconstructing a piecewise constant time series that fits the input. Figure $4 \mathrm{~b}$ represents the temporal evolution of CCD column \#1341 after Step 3. It can be compared with Fig. 4a.

For a selected pixel that ignited in April 2011, Fig. 5 shows the temporal evolution of its variance-stabilized dark signal. The UHT staircase function is overplotted. The prevalent goodness of the UHT fit can be appreciated in this particular case.

We reprogrammed the UHT for the interactive data language (IDL) on the basis of the R package that Fryzlewicz (2007) made available online. A thresholding of the unbalanced Haarlike wavelet coefficients is needed to not completely reconstruct the input, but to instead preserve the significant steps only, and hence to denoise. We defined this with Eq. (9), which was devised empirically to single out the physical signal:

$|w| \times$ scale $^{2.25}>4 \times 10^{4}$

where $w$ is the coefficient of the considered UH wavelet, and scale is the length of the shortest of its two piecewise constant segments. Clearly, this forces short steps to display jumps of large amplitudes, while it permits longer periods of stability to result from smaller leaps, as desired.

To test our ideas, we considered a series of 40 successive data points. They were allowed to constitute a single level as a whole if the amplitude of the step that is needed to explain their evolution is larger than $4 \times 10^{4} / 40^{2.25} \approx 10$. But this amplitude reaches $\sim 100$ for 14 successive points and $\sim 1000$ for only five successive points. The fast and relatively large modulation of a signal that is otherwise flat on the longer scales will not be able to trigger a long-duration level change (because it is essentially flat) or a pattern of successive crenels (because they would not be large enough). Such a time series, which is representative of the RTS of hot pixels, will accordingly be smoothed by our algorithm, even if the variability reaches a few hundreds (see an 
illustration of this in Fig. 5). Note, however, that these amplitudes are measured in the non linear space of the Box-Cox transformed signal.

\subsubsection{Step 4: robust regression for constant dark signal periods}

After identifying the instants of time when a given IZ pixel ignites, or when it changes level if it was already hot, or when any pixel of its corresponding MZ column discloses a significant jump, it becomes possible to separate $\Psi^{\mathrm{MZ}}$ and $\Upsilon^{\mathrm{IZ}}$, the $\mathrm{MZ}$ and IZ dark components, respectively, by means of a linear regression on the integration time. This is the purpose of Step 4, where we considered all dark frames that were acquired with different integration times $T^{\prime}$.

Because the pixel coordinates $(i, j)$ are fixed and $t$ is in a time interval $\Delta$ when $\Psi_{i, j}^{\mathrm{MZ}}(t)$ and $\Upsilon_{i, j}^{\mathrm{IZ}}(t)$ can be supposed constant, Eq. (5) suggests that a linear regression (see e.g. Kutner et al. 2004, as a possible reference) will allow separating the two. The regression must be robust against the heteroscedasticity of the data that cannot be variance-stabilized anymore since we intend to exploit their proportionality to $T^{\prime}$. To this aim, we used a scheme that employs a weighted $\mathrm{L}_{1}$ norm, as defined in Eq. (11) below.

We defined $d s_{\Delta}(t)=d s(t)$ for which $t \in \Delta$. Furthermore, for each of the $K$ distinct $T_{k}^{\prime}$, we gathered the dark signals $d s_{\Delta}^{k}(t)$ defined by the $d s_{\Delta}(t)$ for which $T^{\prime}(t)=T_{k}^{\prime}$ and computed

- their median: $M E D_{\Delta}^{k}=\operatorname{median}_{t}\left(d s_{\Delta}^{k}(t)\right)$,

- their mean absolute deviation with respect to the median: $M A D_{\Delta}^{k}=\operatorname{median}_{t}\left(\left|d s_{\Delta}^{k}(t)-M E D_{\Delta}^{k}\right|\right)$, and

- the maximum of all standard deviations estimated from Eq. (B.3): $M S D_{\Delta}^{k}=\max _{t}\left(G \cdot d s_{\Delta}^{k}(t)+R N^{2}\right)^{0.5}$.

As it is known that $\sigma \approx 1.4826 \times$ MeanAbsDev (Rousseeuw \& Leroy 2005), we defined $\sigma_{\Delta}^{k}=\max \left[M S D_{\Delta}^{k}, 1.4826 \cdot M A D_{\Delta}^{k}\right]$ to tentatively improve the standard deviation estimate for the hotter pixels. A robust linear regression (RLR) was then obtained by solving iteratively

$$
\begin{gathered}
\left(\Upsilon_{\Delta}^{\mathrm{RLR}}, \Psi_{\Delta}^{\mathrm{RLR}}\right)_{i, j}=\underset{\Upsilon>0, \Psi>0}{\arg \min } \mathrm{D}_{1}\left(d s_{\Delta},[\Upsilon, \Psi]\right) \\
\text { with, } \\
\mathrm{D}_{1}\left(d s_{\Delta},[\Upsilon, \Psi]\right)=\frac{1}{K} \sum_{k=1}^{K}\left(\frac{\left|d s_{\Delta}^{k}-\left(\Upsilon \times T_{k}^{\prime}+\Psi\right)\right|}{\sigma_{\Delta}^{k}}\right)
\end{gathered}
$$

For $K=1$, the RLR is still fed with the available $d s_{\Delta}^{k}(t)$, but also with $\Psi_{\text {Previous } \Delta}^{\mathrm{DSM}}$ (the last DSM-computed value for $\Psi$ ), associated with a null integration time $\left(T^{\prime}=0\right)$. This situation occurs. It is mostly a result of the hot pixels RTS noise, which sometimes generates $\Delta$ periods that are too short.

\subsubsection{Step 5: updating and resampling on a daily grid}

We finally need to acknowledge that the intervals $\Delta$ are dissimilar in their capacity to inform about $\left(\Upsilon_{\Delta}, \Psi_{\Delta}\right)_{i, j}$. For this reason, the $\left(\Upsilon_{\Delta}^{\mathrm{RLR}}, \Psi_{\Delta}^{\mathrm{RLR}}\right)$ computed in Step 4 cannot simply be assigned to the period of time covered by $\Delta$. The following updating scheme was used to adequately mix a fresh estimate computed in $\Delta$ by Step 4 , with the previous one, already assigned to Previous $\Delta$.
A quality index $Q_{\Delta,(i, j)}$ was computed for each $\Delta$ :

$$
\begin{aligned}
Q_{1} & =\max _{k} T_{k}^{\prime}-\min _{k} T_{k}^{\prime} \\
Q_{2} & =\sqrt{K} \\
Q_{3} & =\exp \left(-\left|\log \mathrm{D}_{1}\left(d s^{\Delta},\left[\Upsilon^{\Delta}, \Psi^{\Delta}\right]\right)\right|\right) \\
Q_{\Delta} & =Q_{1} \times Q_{2} \times Q_{3}
\end{aligned}
$$

$Q_{1}$ and $Q_{2}$ naturally favor the leverage provided by dissimilar and/or numerous $T_{k}^{\prime}$, respectively. $Q_{3}$ measures both the goodness and the reliability of the Step 4 RLR. Note that $Q_{3}$ reaches a maximum for $\mathrm{D}_{1}\left(d s^{\Delta},\left[\Upsilon^{\Delta}, \Psi^{\Delta}\right]\right)=1$. This is the desirable behavior since the terms of the sum in Eq. (11) must obviously not be much larger than 1 , if the fit is to be good. At the same time, they must not be much smaller than 1 either, as this hints at poor statistics due to a lack of data. In case $K=1, Q_{\Delta}$ takes its previous value, arbitrarily divided by 50 .

Now, $\Upsilon$ and $\Psi$ can be updated by taking the weighted mean of their new and old estimates, with their respective quality index as coefficients. The quality index was also updated, but using the geometric mean.

$$
\begin{aligned}
& \Upsilon_{\Delta}^{\mathrm{DSM}}=\left(Q_{\Delta} \cdot \Upsilon_{\Delta}^{\mathrm{RLR}}+Q_{\text {Previous } \Delta}^{\mathrm{DSM}} \cdot \Upsilon_{\text {Previous } \Delta}^{\mathrm{DSM}}\right) /\left(Q_{\Delta}+Q_{\text {Previous } \Delta}^{\mathrm{DSM}}\right) \\
& \Psi_{\Delta}^{\mathrm{DSM}}=\left(Q_{\Delta} \cdot \Psi_{\Delta}^{\mathrm{RLR}}+Q_{\text {Previous } \Delta}^{\mathrm{DSM}} \cdot \Psi_{\text {Previous } \Delta}^{\mathrm{DSM}}\right) /\left(Q_{\Delta}+Q_{\text {Previous } \Delta}^{\mathrm{DSM}}\right) \\
& Q_{\Delta}^{\mathrm{DSM}}=\sqrt{Q_{\text {Previous } \Delta}^{\mathrm{DSM}} \cdot Q_{\Delta}} .
\end{aligned}
$$

Finally, to provide a straightforward dark signal correction scheme, daily IZ and MZ matrices were computed pixel per pixel by taking the daily median of the updating stage outputs, if any, or by duplicating the values of the day before otherwise. Figure 6 shows the result of Step 5 for CCD column \#1341, the same column as in Fig. 4. Figure 7 shows the outcome of Step 5 for the whole IZ and MZ as of 3 Nov. 2012. Note that at this stage, a binary mask of the hot pixels was additionally constructed by thresholding the IZ cartographies with a value (viz. $50 \mathrm{e}^{-} \mathrm{pxl}^{-1} \mathrm{~s}^{-1}$ ) that is legitimated in Sect. 4.2.

The resulting daily FITS files are used by the L1 processing chains of the PICARD Science Mission Center (CMS-P), and distributed online from the SODISM homepage at LATMOS ${ }^{1}$.

\section{Model examination}

In the present section, the products of the above described model are investigated. We measure in Sect. 4.1 the discrepancy between our DSM and a number of observed dark frames, and we verify that the bias is small. Second, we study in Sect. 4.2 the temporal evolution of the estimated IZ dark current. A few additional verifications are reported at the end of this section.

\subsection{Verifying the dark signal model on dark frames}

The goal is here to inspect the outcome of the model in the situation for which the solution that the DSM should ideally deliver is known. We selected dark frames programmed with the $7.4 \mathrm{~s}$ integration time, that is, the most common configuration. It is the integration time of the images recorded in the $535 \mathrm{H}$ channel that is dedicated to the helioseismologic investigations, which are demanding in terms of photometric correction. This choice also helps to assemble a regular sampling, set weekly hereafter.

All along the mission, the corrected dark signal appears to be tightly distributed around zero, as expected (Fig. 8a). The

http://picard.projet.latmos.ipsl.fr/ 
(a) CCD column \#1341 Image zone DSM evolution

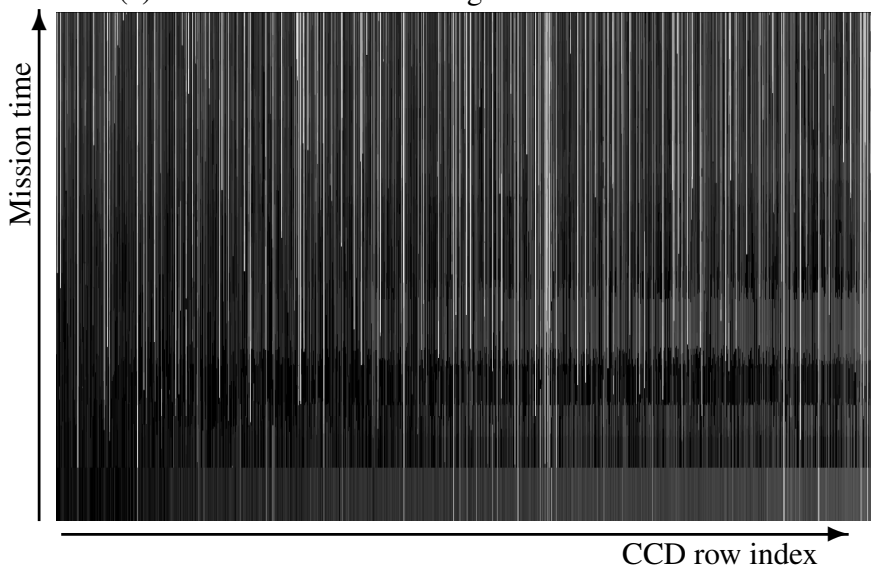

(b) Memory zone DSM evolution for the same column

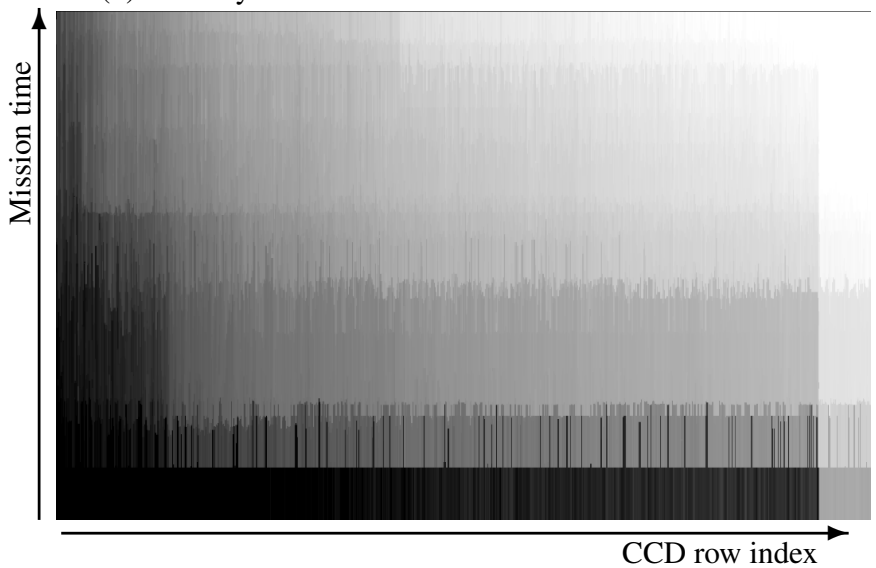

Fig. 6. a) Evolution of the IZ DSM for CCD column (image row) \#1341. The log of the IZ DSM is represented in gray scale, and the information from all successive frames has been stacked vertically. The advent of hot pixels in the IZ appears as bright semi-infinite vertical lines. b) Evolution of the MZ DSM for the same CCD column. The representation conventions are the same as above, but in contrast to panel a), the gray scale is linear. The presence or advent of hot pixels in the MZ is seen as steps that brighten the figure rightward and upward simultaneously. The dark current in the MZ appears as an inclined plane, whose slope increases with time due to the gradual emergence of a distribution of hot pixels in the MZ. For both IZ and MZ, the first weeks (bottom of the images) are only poorly estimated because there were no data to feed the DSM/7.4 for these dates.

few lighter vertical strips are related to frames with many CRHs taken, for example, while crossing the SAA, and obviously, the DSM does not model the CRHs. By fitting a Gaussian function to the histogram (Fig. 8b), we learned that the DSM underestimates the dark signal by $\sim 3.1 \mathrm{ADU} \approx 5 \mathrm{e}^{-}$. The model bias is thus small.

The Gaussian fit also provides the RMS deviation of our DSM. On average from Oct. 2010 till Oct. 2012, the RMSD and the FWHM are 4.7 ADU rms $\approx 25 \mathrm{e}^{-} \mathrm{rms}$ and $34.6 \mathrm{ADU} \approx 60 \mathrm{e}^{-}$, respectively. This is only $50 \%$ more than the read noise (RN), which is in the range of 9-11 ADU rms for the whole period (see Sect. B.2 or Table 1). The observed RMSD accounts for possible algorithmic inaccuracies, and certainly more predominantly for phenomena that cannot be modeled, such as the RTS noise of hot pixels in the memory zone.

A number of outliers depart from the Gaussian behavior. The resulting shoulders are visible in Fig. 8b. By subtracting the (a) Image zone dark current, 3 Nov. 2012

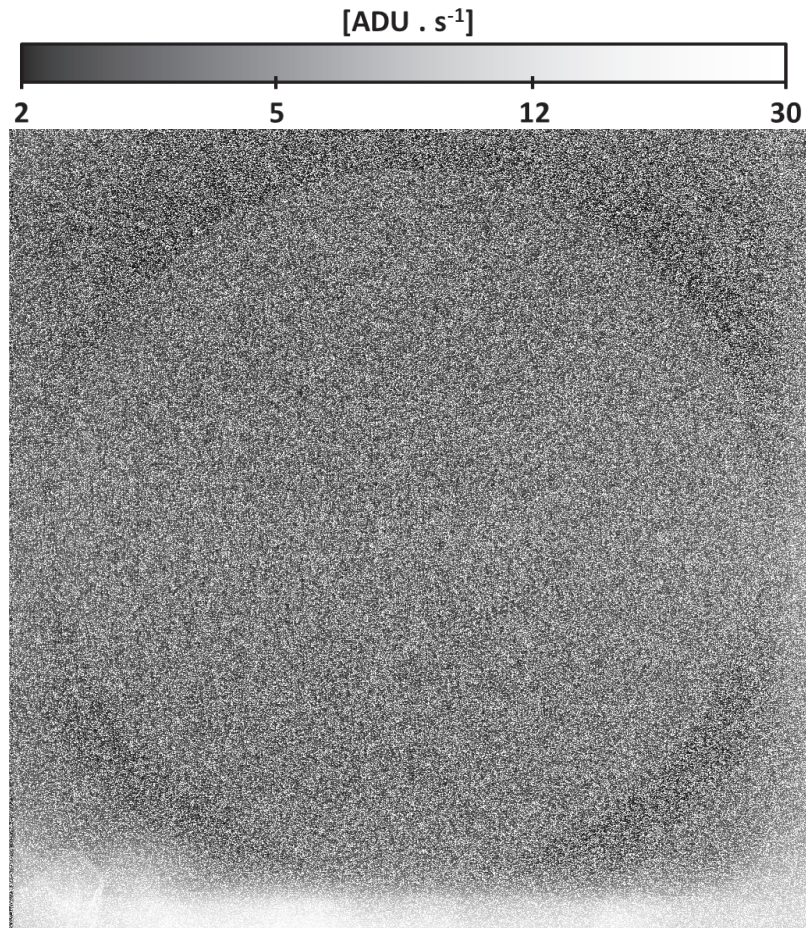

(b) Memory zone dark signal, 3 Nov. 2012

[ADU . $\left.\mathrm{s}^{-1}\right]$

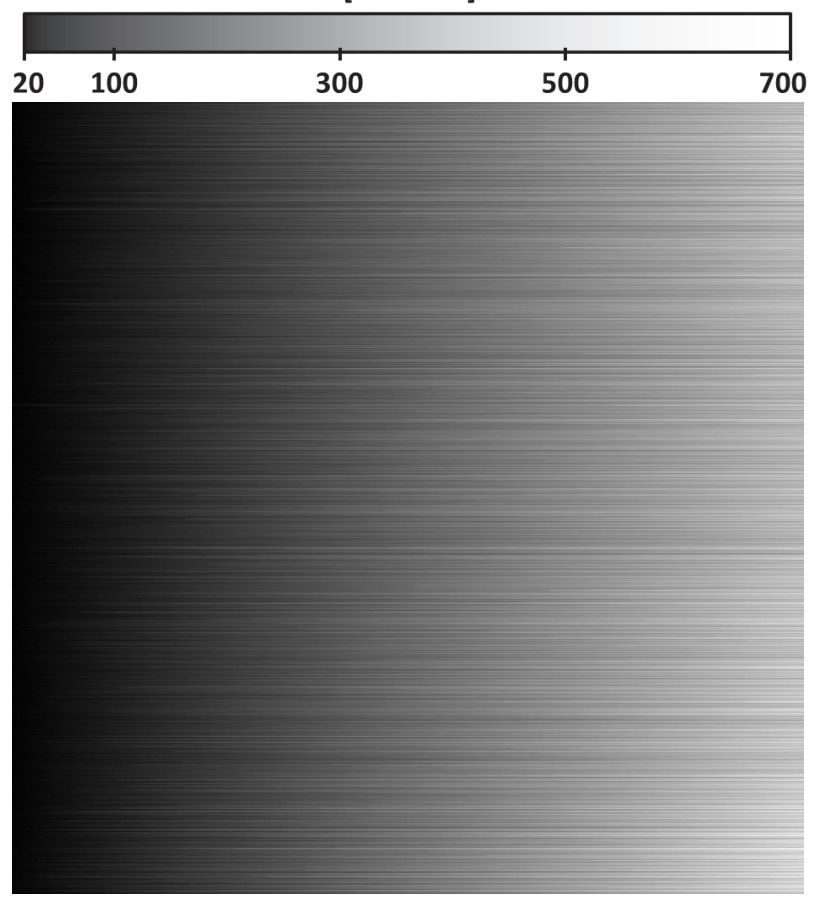

Fig. 7. a) DSM dark current in the image zone as of 3 Nov. 2012. The gray scale is logarithmic. Black corresponds to $2 \mathrm{ADU} / \mathrm{s}$ and white to $30 \mathrm{ADU} / \mathrm{s}$. At the bottom of the image, one can notice an extended contamination by a spurious signal. Its level is in the 6-20 ADU pxl ${ }^{-1} \mathrm{~s}^{-1}$ range (see also Fig. 9). A persistent solar image is also apparent in the IZ dark current. Its amplitude is about $0.2 \mathrm{ADU} \mathrm{pxl}^{-1} \mathrm{~s}^{-1}$. b) Dark signal in the memory zone reconstructed by the DSM as of 3 Nov. 2012. The gray scale is linear and displays the quasi linear increase of the dark signal in MZ (from left to right in this representation). Black corresponds to $20 \mathrm{ADU}$ and white to $700 \mathrm{ADU}$.

above Gaussian fits from the histograms, we estimate the outliers to represent $\sim 5 \%$ of the pixels in late 2010 and $\sim 20 \%$ in 
(a) Evolution of the histograms of $7.4 \mathrm{~s}$ corrected dark frames

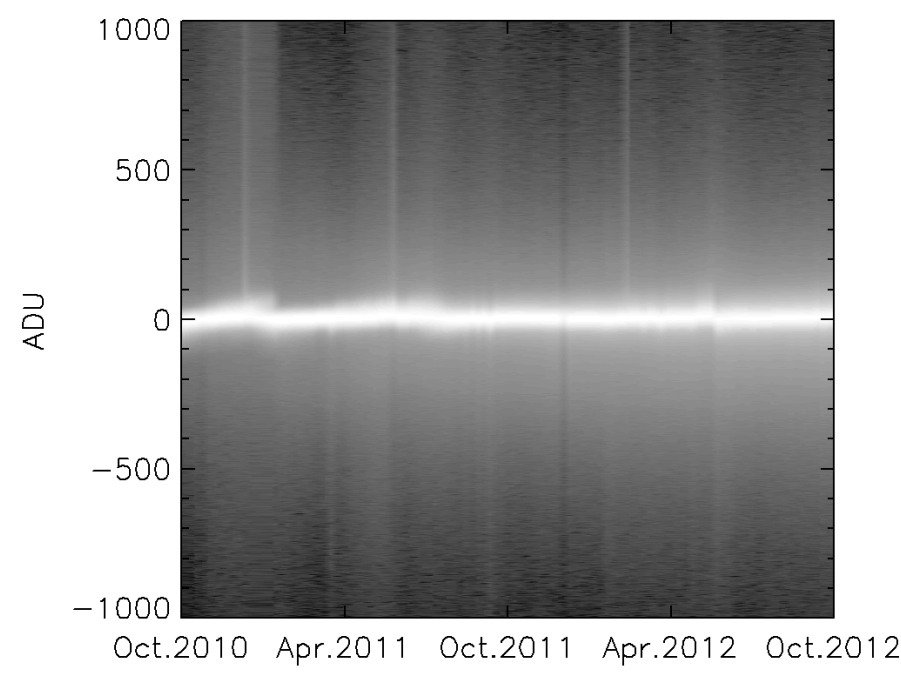

(b) Average histogram and Gaussian fit

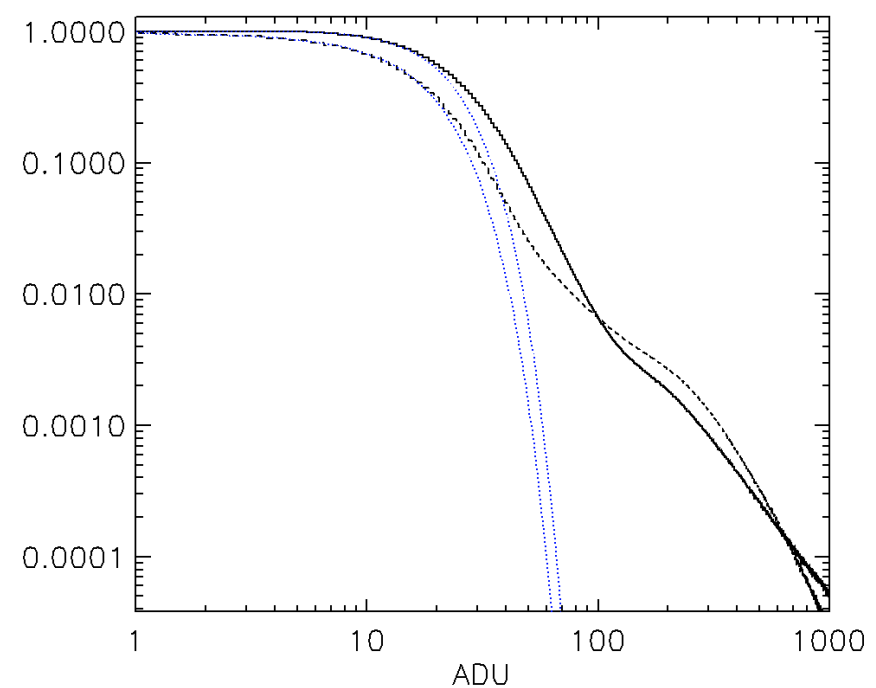

Fig. 8. a) Temporal evolution of corrected dark frame histograms. Dark frames with a weekly regularity and $T^{\prime}=7.4 \mathrm{~s}$ integration time have been selected. Their histogram is coded with a logarithmic gray scale. Most pixels display a corrected signal near zero, as expected. b) Average histogram of all above dark frames. Both axes have log scales. The number of occurrences corresponding to negative signals are represented by the dashed line. The fitted Gaussian function is overplotted with a blue dotted line. Its center (the model bias), its standard deviation (model error), and its full width at half maximum (FWHM) is worth $\sim 3.1$ ADU $\sim 14.7 \mathrm{ADU}$, and $\sim 34.6$ ADU, respectively.

late 2012. These percentages cover all CRHs and hot pixels with non-Poissonian behaviors. They cannot be estimated by a DSM that is based on the assumption of a sufficiently prolonged constancy. The error can be positive or negative. It reaches several thousand ADU, but this level concerns a minute fraction of the pixels. The solution is to flag the hot IZ pixels to exclude them from the subsequent scientific exploitation.

Note that columns containing hot MZ pixels cannot be excluded altogether since this would then be the case of every column. We must consequently rely on the fact that their relatively unpredictable dark contributions should compensate mutually.

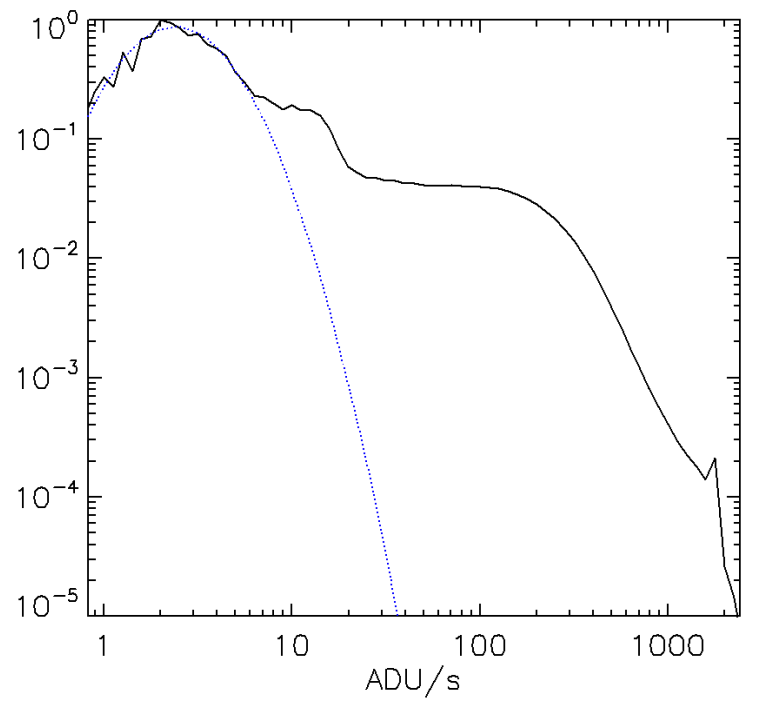

Fig. 9. Normalized histogram of the Jul. 2010-Nov. 2012 maps reconstructed by the dark signal model for the image zone. The histogram mode represents the cool pixels. It can be fitted by a lognormal function (blue dotted line), which gives 2.39 ADU $\mathrm{pxl}^{-1} \mathrm{~s}^{-1} \approx 4.1 \mathrm{e}^{-} \mathrm{pxl}^{-1} \mathrm{~s}^{-1}$. The first small shoulder around 6-20 ADU $\mathrm{pxl}^{-1} \mathrm{~s}^{-1}$ comprises the pixels that are polluted by some spurious signal in the bottom of the image. The second shoulder describes the hot pixel dark current distribution. It appears to be flat from 10-20 ADU pxl $l^{-1} \mathrm{~s}^{-1}$ to $200 \mathrm{ADU} \mathrm{pxl}^{-1} \mathrm{~s}^{-1}$. The hottest pixels deliver $2000 \mathrm{ADU} \mathrm{pxl}{ }^{-1} \mathrm{~s}^{-1} \approx 3.5 \mathrm{ke}^{-} \mathrm{pxl}^{-1} \mathrm{~s}^{-1}$.

However, as mentioned, this leads to a noise that probably dominates the RMSD.

\subsection{Evolution of the dark current distribution in $I Z$}

The normalized histogram of all DSM maps for IZ is plotted in Fig. 9. Its peak corresponds to the cool pixels. Fitting a lognormal distribution (Baer 2006), and looking at its mode gives 2.39 ADU pxl ${ }^{-1} \mathrm{~s}^{-1}$ for CPDC, the cool pixel dark current. Using Eq. (B.5), $C P D C=2.39 \mathrm{ADU} \mathrm{pxl}^{-1} \mathrm{~s}^{-1} \times 1.685 \mathrm{e}^{-} / \mathrm{ADU}=$ $4.0 \mathrm{e}^{-} \mathrm{pxl}^{-1} \mathrm{~s}^{-1}$. This is very close and indeed in between the two estimates made in Sect. 2.2.2, namely 3.6 and $4.4 \mathrm{e}^{-} \mathrm{pxl}^{-1} \mathrm{~s}^{-1}$ at $-7.2{ }^{\circ} \mathrm{C}$. We remark that the persistent solar imprint visible in Fig. $7 \mathrm{a}$ is not observed in the histogram. It is indeed on the order of $0.2 \mathrm{ADU} \mathrm{pxl} \mathrm{P}^{-1} \mathrm{~s}^{-1}$ and is therefore merged in the main mode of the histogram.

A special observing campaign was conducted on 29 June 2011 to understand this phenomenon of persistence. It is not reported fully here because the analysis has not been fully conclusive and the effect is weak. However, we summarize the main observations:

1. The solar imprint is weak $\left(0.2 \mathrm{ADU} \mathrm{pxl}^{-1} \mathrm{~s}^{-1}\right)$, and independent of the wavelength of the previous solar image and on its exposure time.

2. In contrast, the dark current from pixels that were not illuminated is a slight function of the exposure time and of the wavelength of the previous solar image!

3. These two components decrease slowly with time under dark conditions.

These observations are rather unexpected. One tentative interpretation suggests that a small heating of the CCD and of the optical filters ahead is responsible. The heating would be generated by the opening of the mechanical shutter. 


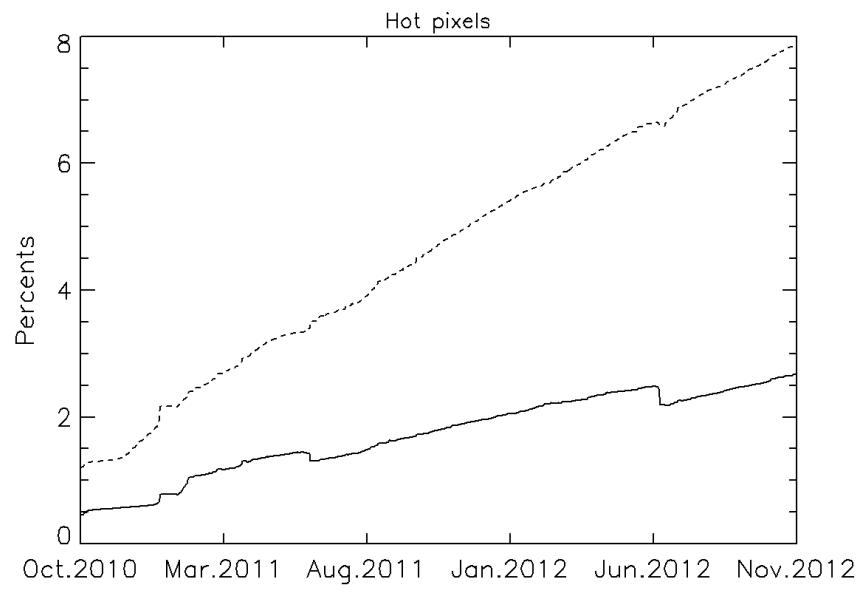

Fig. 10. Temporal evolution of the fractional area covered by hot pixels with respect to the whole image zone. The solid line represents hot pixels delivering more than $250 \mathrm{e}^{-} \mathrm{pxl}^{-1} \mathrm{~s}^{-1}$, while the dashed line corresponds to warm pixels delivering from 50 to $250 \mathrm{e}^{-} \mathrm{pxl}^{-1} \mathrm{~s}^{-1}$. Note the two sudden drops in the otherwise gradual growth of the number of hotter pixels. They occur in mid-June 2011 and mid-June 2012, when 2-3 day bakeouts took place.

The first shoulder of the distribution, at about 6-20 ADU pxl ${ }^{-1} \mathrm{~s}^{-1}$, corresponds to a pollution by an unknown signal near the image bottom, as pointed out in the caption of Fig. 7a.

The second shoulder is flat and extends from $30 \mathrm{ADU} \mathrm{pxl} \mathrm{A}^{-1} \mathrm{~s}^{-1}$ to $\sim 2000 \mathrm{ADU} \mathrm{pxl}^{-1} \mathrm{~s}^{-1} \approx 3400 \mathrm{e}^{-} \mathrm{pxl}^{-1} \mathrm{~s}^{-1}$. This plateau represents the detectable hot pixels, whose dark current values is quasi equiprobable over at least one order of magnitude. This study allows defining a threshold at $30 \mathrm{ADU} \mathrm{pxl}^{-1} \mathrm{~s}^{-1}$, viz. $\sim 50 \mathrm{e}^{-} \mathrm{pxl}^{-1} \mathrm{~s}^{-1}$, for flagging the hot pixels in the image zone. Indeed, this value belongs to the second shoulder and not to the first one. Thus, the $30 \mathrm{ADU} \mathrm{pxl}^{-1} \mathrm{~s}^{-1}$ threshold identifies hot pixels with no risk of erroneously triggering in the bottom part of the image that is contaminated by another spurious signal in excess. The hottest pixels have a dark current a hundred times lower than specified by the manufacturer $\left(3400 \mathrm{e}^{-} \mathrm{pxl}^{-1} \mathrm{~s}^{-1}\right.$ instead of $300 \mathrm{ke}^{-} \mathrm{pxl}^{-1} \mathrm{~s}^{-1}$, see Sect. 2.2.2)

We now investigate the temporal evolution of the hot pixels distribution. We split their severity into two separate ranges. The lower range extended from the limit of hot pixel detectivity $\left(50 \mathrm{e}^{-} \mathrm{pxl}^{-1} \mathrm{~s}^{-1}\right)$ to $\theta=250 \mathrm{e}^{-} \mathrm{pxl}^{-1} \mathrm{~s}^{-1}$. The upper range contained the hotter pixels, which delivered more dark current than $\theta$. The value of $\theta$ was chosen to show the annealing threshold effect discussed below. The evolution of both percentages is plotted in Fig. 10.

For the lower range, the rate of hot pixel ignition slightly decreased over the mission lifetime, and worth $\sim 350$ new hot pixels per day on average, viz. $\sim 3 \%$ of the CCD image zone per year. For the higher range, it is constant and worth $\sim 150$ new hot pixels per day, viz. $\sim 1.2 \%$ of the CCD image zone per year. This makes a total of $\sim 500$ new hot pixels per day, which is very similar to the 1000 new hot pixels per day measured by Polidan et al. (2004) and Baggett et al. (2012) for a CCD with twice the SODISM format. Their HST/WFC3 UVIS experiment used CCDs of the E2V 43 series, whose format is $2048 \times 4096$, and whose pixel size is $15 \mu \mathrm{m}$ (instead of $13.5 \mu \mathrm{m}$ in our case). Like SODISM's, these CCDs have a buried channel, an MPP implant, and are backthinned.
We additionally remark that the $50 \mathrm{e}^{-} \mathrm{pxl}^{-1} \mathrm{~s}^{-1}$ criterion leads to a $\sim 10 \%$ area coverage by hot pixels in late 2012, while we found $20 \%$ pixels departing from the Gaussian behavior in the previous section. This is because many of them fall below our threshold (see Fig. 9). But lowering it would flag too many pixels as hot, among which many would moreover become false positives.

The drops in the slope observed in mid-June 2011 and midJune 2012 in Fig. 10, especially for the hotter pixel curve, are coincident with the two SODISM bakeout operations. Indeed, they reveal that the pixels with a dark current higher than $\theta$ were instantaneously (but only partially) annealed on these occasions. The faster annealing of hotter pixels as compared to the annealing of the "warm" ones has been noted by Polidan et al. (2004) and studied by Marshall et al. (2005). An interesting possibility inferred from the latter reference is that the hotter pixels of SODISM may be to some extent constantly self-annealing due to the lukecold (cool, but not very cold) operating temperature of its CCD.

Some more verifications were made. The average dark current of the whole image zone (including the hot pixels contribution) was found to increase from a few $\mathrm{e}^{-} \mathrm{pxl}^{-1} \mathrm{~s}^{-1}$ in mid2010 up to $30 \mathrm{e}^{-} \mathrm{pxl}^{-1} \mathrm{~s}^{-1}$ in late 2012. This can be compared with the equivalent estimation that can be made for the memory zone. Interestingly, the integrated dark current in the $\mathrm{MZ}$ was found to be consistently $20 \%$ higher than the IZ counterpart. This could mean that the shield of the MZ favors the generation of non ionizing collisions at the origin of displacement damages and hence, of hot pixels.

Finally, it was established that the mean level of the first dark row of the MZ increases gradually from $\sim 0 \mathrm{e}^{-} / \mathrm{pxl}$ in mid-2010 up to $\sim 40 \mathrm{e}^{-} / \mathrm{pxl}$ in late 2012 . This is possibly due to surface dark current seeping increasingly into the pixels during the frametransfer phase.

\section{Summary and conclusions}

The first part of our conclusion summarizes the information gathered about the PICARD-SODISM CCD camera. The second part concludes about our modeling of the dark signal. The third and the fourth parts recapitulate more general considerations derived from our study.

\subsection{SODISM camera}

The inverse of the PICARD-SODISM camera gain is $G_{16}^{-1} \approx$ $0.85 \mathrm{e}^{-} / \mathrm{ADU}_{16}$ bit when the image is coded on 16 bits, and $G_{15}^{-1} \approx 1.70 \mathrm{e}^{-} / \mathrm{ADU}_{15}$ bit when it is coded on 15 bits, as is more commonly the case. These values have been obtained from flight data by means of the photon transfer technique (Janesick et al. 1987b) applied to a special sequence of dark frames recorded in July 2010. The overall camera read noise is measured to be $15 \mathrm{e}^{-}$rms in July 2010 and $20 \mathrm{e}^{-}$rms in late 2012. This is four and five times higher, respectively, than the intrinsic read noise of the CCD. The camera read noise is contaminated by an orbital variability.

\subsection{Dark signal model and its application to SODISM CCD}

At the operating temperature of the Flight $\operatorname{CCD}\left(-7.2^{\circ} \mathrm{C}\right)$, the dark current of its non hot pixels is predicted to be either 3.6 or $4.4 \mathrm{e}^{-} \mathrm{pxl}^{-1} \mathrm{~s}^{-1}$, depending on the assumed model. 
It is possible to correct for the dark signal of frame-transfer CCDs by applying the method presented in this paper. The dark signal components of the CCD image zone and memory zone have been estimated daily by our DSM. Its results are available online and exploited by SODISM Level 1 products.

At $-7.2{ }^{\circ} \mathrm{C}$, the dark current of non hot pixels is estimated to be $4.0 \mathrm{e}^{-} \mathrm{pxl}^{-1} \mathrm{~s}^{-1}$ in the image zone (in good agreement with the previously reported expectation), and the hottest dark pixels produce no more than $3.5 \mathrm{ke}^{-} \mathrm{pxl}^{-1} \mathrm{~s}^{-1}$.

Our DSM exhibits a global bias of $\sim 5 \mathrm{e}^{-}$and an rms deviation of $25 \mathrm{e}^{-} \mathrm{rms}$ (for images taken with a $7.4 \mathrm{~s}$ integration time). A threshold can be reliably set at $50 \mathrm{e}^{-} \mathrm{pxl}^{-1} \mathrm{~s}^{-1}$ to flag the hot pixels of the image zone for their subsequent optional dismissal by science investigations. With this threshold, $\sim 1.5 \%$ of the Flight CCD pixels were declared hot at commissioning in Oct. 2010, and $\sim 11 \%$ in Dec. 2012.

\subsection{Scientific use of CCDs}

For the use of CCDs with a frame-transfer architecture, we have verified that the correction of their dark signal is complicated, especially when their temperature is not cold enough or when the readout is not fast enough to suppress the dark signal contribution from the memory zone.

We suggest to plan flight operations that regularly cycle integration time across its admissible range. In view of the need of future DSMs, dark frame programming should include such cycling.

Really cold operations are desirable for frame-transfer CCD operations, although "lukecold" (mildly cool) temperatures may mitigate CTE problems and the growth rate of hot pixels.

Frame-transfer CCDs should therefore be employed when their advantages $(\sim 100 \%$ observational duty cycle, avoidance of a mechanical shutter) surpass their inconveniences.

Bakeout operations at room temperature for a few days do anneal a fraction of the hot pixels, particularly the hotter ones, but dedicating a few days to this, instead of observing, can be considered worthless with respect to their limited effectiveness. Alternatively, programming many more bakeouts might maintain the CCD near its pristine state.

\subsection{Unbalanced Haar transform}

Aspects of our method can be useful to other applications. Modeling the dark signal of a full-frame CCD would be a natural application, similar to the present one, but without the need to regress.

The unbalanced Haar transform of Fryzlewicz (2007), or adapted versions thereof, can be particularly powerful to fit piecewise constant functions in various situations.

Acknowledgements. PICARD-SODISM is a project supported by the Centre National d'Études Spatiales (CNES), the CNRS Institut National des Sciences de l'Univers (INSU), and the Belgian Space Policy (BELSPO). The SODISM instrument has been designed and built in collaboration between CNRS-LATMOS and CNES. It is operated in coordination by the Scientific Mission Center (CMS-P) at BUSOC, Belgium, the Centre de Contrôle (CCC) at the CNES, and the LATMOS. The authors acknowledge the work of Michel Rouzé, Florence Duchevet, and their teams at the CNES, and of Claudio Queirolo, Anuschka Helderweirt, and Dirk Pauwels at BUSOC for their modification of the in-flight payload operations, as well as of Abdenour Irbah, Momar Cissé, Marc Lin, and Christophe Dufour at the LATMOS for their development of the SODISM processing chain and for their support. The authors would like to also thank Peter Maggs and David Morris from E2V, and Pernelle Bernardi, Didier
Tiphène, Vincent Lapeyrere, and Jean Tristan Buey from LESIA for their answers to our questions. J.-F. H. thanks Francis Dalaudier, Véronique Delouille, and Thierry Corbard for useful suggestions. J.-F. H. has also appreciated the help of Caroline Guérin, André-Jean Vieau, Yann Delcambre, and Olivier Thauvin of the LATMOS for their kind support to the work that is reported here. The authors acknowledge gratefully the useful suggestions made by an anonymous referee.

\section{Appendix A: CCD procurement and characterization by the CoRoT program}

The SODISM CCDs are devices from the CCD 42-80 series of E2V. They have been purchased in 2001 as part of the procurement of a batch of ten devices for the CoRoT astrometric space mission (Auvergne et al. 2009), which has been developed a few years before PICARD and was also managed by the French Space Agency (CNES). The main reason for selecting a device of the 42-80 series for SODISM has been the similarity of the specifications and the project synergy with the CoRoT CCD program at the Laboratoire d'Études Spatiales et d'Instrumentation en Astrophysique (LESIA; Bernardi et al. 2004; Buey et al. 2005; Lapeyrere et al. 2006; Gilard et al. 2008, 2010).

To enhance their quantum efficiency (QE) the CCD 42-80 sensors are backthinned. This technology improves the sensitivity, especially in the mid-ultraviolet (MUV), near-ultraviolet (NUV) and blue spectral ranges. QE and pixel response non uniformity (PRNU) are not reported or discussed in more detail as these issues are beyond the scope of the present paper.

The four CoRoT flight CCDs were selected on the basis of an extensive series of tests carried out by the CoRoT CCD program (Bernardi et al. 2004; Lapeyrere et al. 2006).

Five CoRoT devices that had passed the above screening became available to the SODISM project, who additionally procured two more CCDs from E2V with identical specifications to those of the former batch (see Table A.1). Using the result of the CoRoT measurements and some additional characterization, the SODISM project selected for flight the CCD 42-80-1-985 \#60 (9271-18-08), referred to simply as CCD\#60, or as SODISM flight CCD.

Table A.1. List of the CCDs available to the SODISM project.

\begin{tabular}{llc}
\hline \hline Project origin & Reference & Name \\
\hline SODISM & $9274-03-06, \# 78$ & N/A \\
SODISM & $9274-18-06, \# 89$ & N/A \\
CoRoT & $9271-12-07, \# 55$ & Amon \\
CoRoT & $\mathbf{9 2 7 1 - 1 8 - 0 8 , \# 6 0}$ & Mehen \\
CoRoT & $9235-03-07, \# 62$ & Weneg \\
CoRoT & $9235-15-06, \# 67$ & Apopis \\
CoRoT & $9272-08-07, \# 71$ & Iah \\
\hline
\end{tabular}

Notes. CCD\#60 - the device selected for the SODISM flight instrument - is highlighted.

For CCD\#60, E2V indicated that its vertical CTE was better than 0.999999, that is, CTI $=1-\mathrm{CTE} \leq 10^{-6}$ at beginning of life (BOL), and that up to $\sim 12$ lines of the IZ might be covered by the storeshield of the MZ. The LESIA tests showed that the saturation level of CCD\#60 reach from $7710^{3} \mathrm{e}^{-}$to $11110^{3} \mathrm{e}^{-}$ from the center of the image zone to its edges. This was interpreted as an effect of the imperfect propagation of the electric potential to the electrodes at the middle of the CCD. 


\section{Appendix B: SODISM camera electronics}

\section{B.1. Serial register and readout ports}

The serial register of the E2V 42-80 CCD is split in half. This allows reading out the signal at the two corresponding ports. During nominal SODISM operations, the CCD rows are aligned with the polar axis of the Sun (see Fig. 3). In this configuration, the north pole of the Sun is in the upper half of the image, which corresponds to the right port of the CCD. The lower half of the image is then readout through the left port.

The CCD 42-80 output amplifier is designed to give very good noise performance at low readout rates. In the datasheet, E2V indicates a typical read noise $(\mathrm{RN})$ of $3 \mathrm{e}^{-} \mathrm{rms}$ (with a maximum of $4 \mathrm{e}^{-} \mathrm{rms}$ ) at $20 \mathrm{kHz}$ using correlated double sampling (CDS), and a typical amplifier gain of $4.5 \mu \mathrm{V} / \mathrm{e}^{-}$(possibly ranging from 3 to $6 \mu \mathrm{V} / \mathrm{e}^{-}$).

For CCD\#60, prior to delivery, E2V measured an RN of 3.9 and $4.1 \mathrm{e}^{-} \mathrm{rms}$ for its left and right port, respectively, and they indicated that the amplifier gains were equal to $3.96 \mu \mathrm{V} / \mathrm{e}^{-}$for the left port, and to $3.85 \mu \mathrm{V} / \mathrm{e}^{-}$for the right port, that is, a $2.9 \%$ difference in favor of the left port.

In 2003, these gains were measured by LESIA in the course of the CoRoT screening process, and they were found to be equal to 4.18 and $4.06 \mu \mathrm{V} / \mathrm{e}^{-}$at $-40{ }^{\circ} \mathrm{C}$ (a $3.0 \%$ difference in favor of the left port), with a temperature dependence measured to be $-820 \mathrm{ppm} / \mathrm{K}$ (Bernardi et al. 2004, Table 1). They were later reevaluated at $-40{ }^{\circ} \mathrm{C}$ again (Lapeyrere et al. 2006), and found to amount to 4.33 and $4.24 \mu \mathrm{V} / \mathrm{e}^{-}$, respectively (i.e. a $2.1 \%$ difference in favor of the left port).

Following this latest result, but using the temperature dependence found by Bernardi et al. (2004), the CCD\#60 gains are expected to be worth 4.22 and $4.14 \mu \mathrm{V} / \mathrm{e}^{-}$, respectively, at $-7.2{ }^{\circ} \mathrm{C}$, with a discrepancy of $\sim 2 \%$ in favor of the left port. The exact value of the gain is also a function of the bias voltages, and particularly of $V_{\mathrm{OD}}$ (the output drain), but such extra precision is not required in the sequel.

The saturation level being lower than $111 \mathrm{ke}^{-}$(Sect. A), the $\mathrm{CCD}$ output voltage is thus within the $[0,500] \mathrm{mV}$ range.

\section{B.2. Biasing and sequencing the CCD}

The first of the three main functions of a camera electronics is to supply the CCD with various bias voltages. The reset drain voltage $\left(V_{\mathrm{RD}}\right)$ was set at $8.42 \mathrm{~V}$. The substrate voltage $\left(V_{\mathrm{SS}}\right)$ was set at $0.0 \mathrm{~V}$. The output gate voltages $\left(V_{\mathrm{OG} 1} \& V_{\mathrm{OG} 2}\right)$ were set at $-6.60 \mathrm{~V}$ and $-5.51 \mathrm{~V}$, respectively. The output drain $\left(V_{\mathrm{OD}}\right)$ and the dump drain $\left(V_{\mathrm{DD}}\right)$ voltages were set at $21.78 \mathrm{~V}$ and $14.18 \mathrm{~V}$, respectively.

Second, the camera must sequence the CCD and the shutter mechanism with the needed clocks. We note that the exposure defined by the mechanical shutter occurs strictly within the duration of the electronic integration, which always exceeds the former by $0.4 \mathrm{~s}$ for this reason. Therefore, the integration of dark signal lasts longer than the integration of photo-electrons from the solar exposure by $400 \mathrm{~ms}$. The shutter exposure duration can be programmed to take any value between $0.5 \mathrm{~s}$ (minimum) and $16.0 \mathrm{~s}$ (maximum), by increments of $0.1 \mathrm{~s}$, and the integration time lasts correspondingly from $0.9 \mathrm{~s}$ to $16.4 \mathrm{~s}$. In the header of SODISM FITS files, the shutter exposure duration is given by the TPS_POSE keyword, and the electronic integration duration is provided by the CCD_EXP keyword.

After the integration, the signal that has accumulated in the image zone is transferred to the memory zone in $370 \mathrm{~ms}$. This is

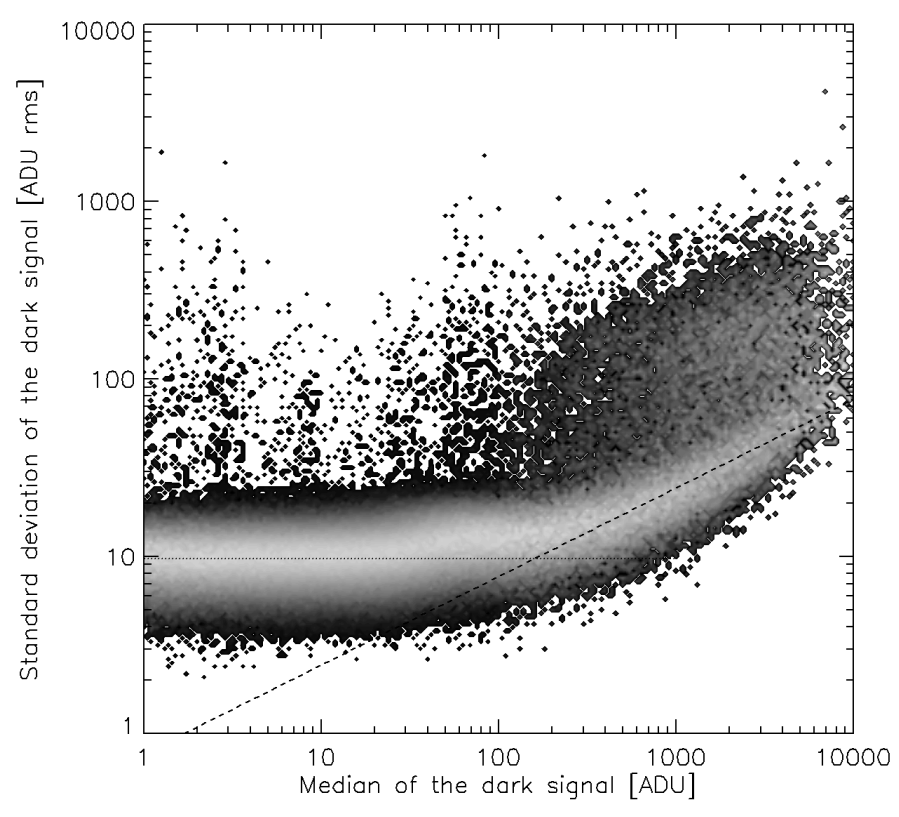

Fig. B.1. Dark signal transfer plot. The median and standard deviation are computed for every pixel of a series of ten dark frames acquired on 31 July 2010 with $T_{i}=16.4 \mathrm{~s}$ and represented here as a twodimensional histogram. A region on the left is dominated by the read noise, which amounts to about $10 \mathrm{ADU}_{15}$ bit rms. The shot-noise dominated region has a slope of 0.5 in the logarithmic representation. Above it, a significant proportion of pixels exhibit ten times more variability than shot noise. These are the hot pixels that suffer from RTS noise (cf. Sect. 1.3).

the frame transfer, and it occurs with closed shutter, viz. under dark conditions. Note that at this point, CTE problems can occur, as well as some evolving contribution from surface dark current, which might add a column-dependent pedestal.

\section{B.3. Pre-flight estimation of the overall gain of the readout chain}

As a third camera function, the electronics amplifies and converts the $0-500 \mathrm{mV}$ analog signals that must be read out from the two output ports of the CCD (see Sect. B.1) into a stream of digital numbers. The conversion cadence being $100 \mathrm{kHz}$ pixel, the CCD line rate is $11.05 \mathrm{~ms}$ per row, and the total readout duration amounts to $22 \mathrm{~s}$ for a whole frame that is read from the two CCD ports simultaneously. The digital stream is then directed to the PICARD Gestion Charge Utile (PGCU) computing unit, where the image is formed, processed, and most often, compressed.

The gain of the preamplifier stage was fixed to $9 \mathrm{~V} / \mathrm{V}$. This is attenuated by the impedance adaptation by a factor 0.94 , which leads to an effective gain of $8.46 \mathrm{~V} / \mathrm{V}$. The unipolar input range of the analog-to-digital converter (ADC) is $0-2.8 \mathrm{~V}$ and it operates on 16 bit. This implies a conversion factor of $2^{16} / 2.8$ analog-todigital unit (ADU) per Volt, that is, $2.34 \times 10^{-2} \mathrm{ADU} / \mu \mathrm{V}$. Hence, the overall gain of the readout chain should be

$$
\begin{aligned}
G_{16} & \approx 2.3410^{-2} \mathrm{ADU} / \mu \mathrm{V} \times 8.46 \times 4.2 \mu \mathrm{V} / \mathrm{e}^{-} \\
& \approx 0.83 \mathrm{ADU}_{16 \mathrm{bit}} / \mathrm{e}^{-}, \\
\text {i.e. } G_{16}^{-1} & \approx 1.20 \mathrm{e}^{-} / \mathrm{ADU}_{16 \mathrm{bit}},
\end{aligned}
$$

with a discrepancy of about $2 \%$ between the two CCD ports. However, most SODISM data are coded on 15 bits, the sixteenth 


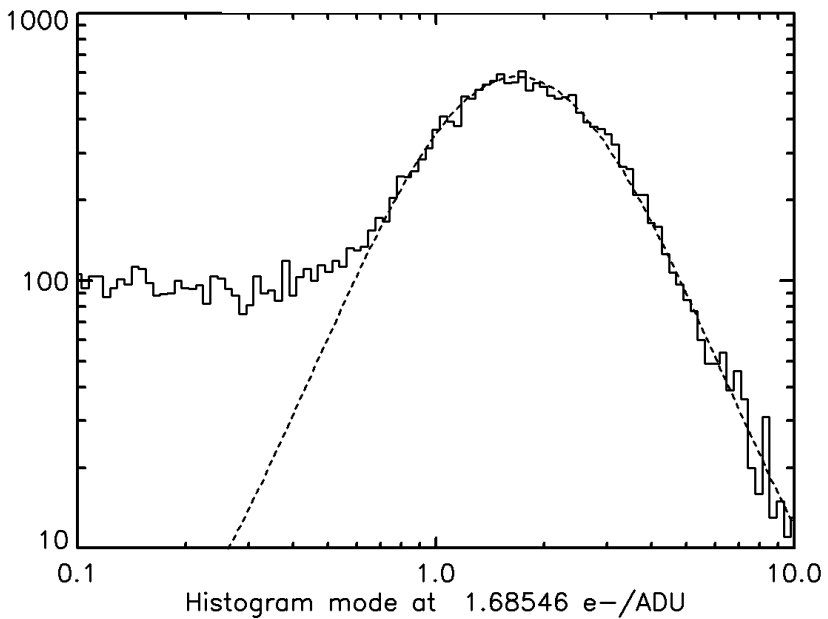

Fig. B.2. Histogram of $\mathrm{G}_{15 \mathrm{bit}}^{-1}$, the inverse of the video gain. Each pixel in the shot-noise-dominated region of Fig. B.1 provides an estimate of $\mathrm{G}_{15 \mathrm{bit}}^{-1}$. A Gaussian function is fitted and gives $1.685 \pm 0.1 \mathrm{e}^{-} / \mathrm{ADU}_{15}$ bit for the mode.

and least significant bit (LSB) is simply dropped. The gain is then halved and becomes

$$
\begin{aligned}
G_{15} & \approx 0.42 \mathrm{ADU}_{15 \mathrm{bit}} / \mathrm{e}^{-}, \\
\text {i.e. } G_{15}^{-1} & \approx 2.40 \mathrm{e}^{-} / \mathrm{ADU}_{15 \mathrm{bit}} .
\end{aligned}
$$

To verify the above bottom-up estimation of the gain, we estimated it by means of the photon transfer technique (Janesick et al. 1987b; Downing et al. 2006) or, more precisely, by using a "dark signal transfer" technique. It states that the random variables of the dark signal DS, of its standard deviation $\sigma_{\mathrm{DS}}$, and of the read noise $\mathrm{RN}$ are related by

$$
\sigma_{\mathrm{DS}}[\mathrm{ADU}]^{2}=G\left[\mathrm{ADU} / \mathrm{e}^{-}\right] \times D S[\mathrm{ADU}]+R N[\mathrm{ADU}]^{2} .
$$

Obviously, this is valid if the noise sources are solely shot noise and read noise. It does not apply to the hot pixels that exhibit erratic fluctuations and intermittency. When RN becomes negligible with respect to the dark Poisson noise, Eq. (B.3) becomes

$$
\begin{aligned}
G^{-1}\left[\mathrm{e}^{-} / \mathrm{ADU}\right] & \approx D S / \sigma_{D S}^{2} \text { or, } \\
\log G^{-1} & \approx \log D S-2 \times \log \sigma_{D S} .
\end{aligned}
$$

\section{B.4. In-flight estimation of the overall gain of the readout chain}

On 31 July 2010, ten dark full frames were acquired on 15 bits, with a $16.4 \mathrm{~s}$ integration time, and within a few hours. For each pixel, after subtracting the offsets, we computed the median and the standard deviation and plotted their two-dimensional histogram (Fig. B.1). The read-noise dominated region and the shot-noise dominated region are clearly distinguishable.

According to Eq. (B.4), $\log G_{15}^{-1}$ can therefore be estimated by measuring the mode of the histogram of [log (Median) $2 \log \sigma]$ (Fig. B.2). Fitting a Gaussian function to this distribution gives

$$
\begin{aligned}
G_{15} & \approx 0.593 \mathrm{ADU}_{15 \mathrm{bit}} / \mathrm{e}^{-}, \\
\text {i.e. } G_{15}^{-1} & \approx 1.685 \mathrm{e}^{-} / \mathrm{ADU}_{15 \mathrm{bit}} .
\end{aligned}
$$

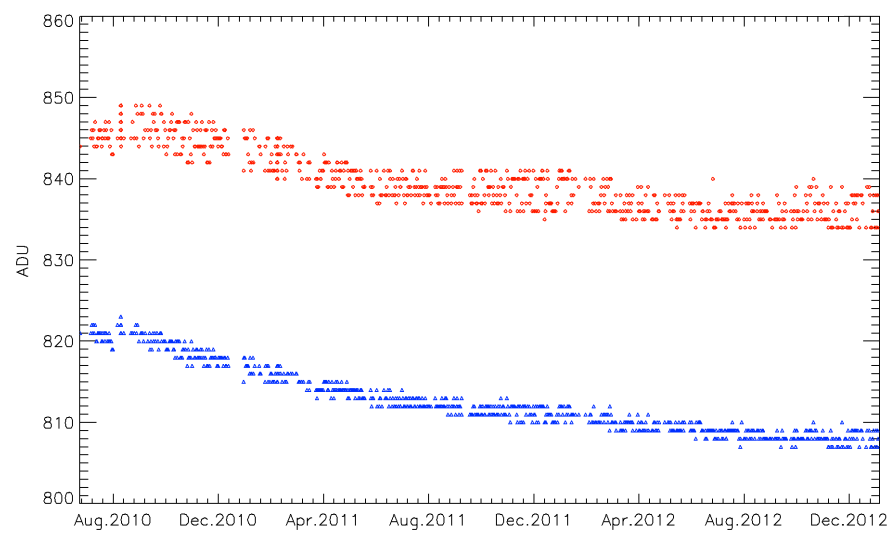

Fig. B.3. Temporal evolution of the offsets of the left port (red diamonds) and right port (blue triangles) of SODISM CCD from July 2010 to December 2012. The offset value is higher and the spread is broader for the upper time series, which corresponds to the left port.

This is hardly consistent with the bottom-up estimate of $2.40 \mathrm{e}^{-} / \mathrm{ADU}_{15}$ bit in Eq. (B.2). The discrepancy may be explained by the approximate knowledge regarding, for instance, the impedances used in the bottom-up approach. We will henceforth use the values of the gain measured by our in-flight analysis, which also provides an estimate for the readout noise, worth 9.7 $\mathrm{ADU}_{15 \text { bit }}$, that is $\sim 16 \mathrm{e}^{-} \mathrm{rms}$, in July 2010 , at BOL. This is about four times higher than the $4 \mathrm{e}^{-} \mathrm{rms}$ value expected from the CCD alone (Sect. B.1).

\section{B.5. Offset bias}

To avoid negative values that the ADC would not be able to convert, a voltage bias was added to the signal before it was fed to the ADC. Resulting offset values were estimated onboard per image (and not per line), by calculating the average of an underscan area (underscan pixels are readout from a given CCD port without having fed it with physical signal, that is, without having clocked the serial register.). These values are in the [840-850] $\mathrm{ADU}_{15}$ bit range for the left port of the CCD, and in the [810-820] ADU $_{15 \text { bit }}$ range for its right port (Fig. B.3). They are later rounded to integer type (unfortunately), transmitted in the telemetry (TM), and appended to the FITS header of the L0 image products (OFFSET_G and OFFSET_D keywords, for the left and the right port, respectively). We notice in Fig. B.3 that the time series of the offsets exhibit some spread and we have demonstrated (not shown in the present paper) that this variability is dominated by an orbital modulation. This coupling is related to the observed read noise excess noted above.

Because the standard deviations of the same underscan area are also computed (and also rounded) onboard, it allows estimating the read noise, which increases from $\sim 9 \mathrm{ADU}_{15 \mathrm{bit}} \mathrm{rms} \approx$ $15 \mathrm{e}^{-} \mathrm{rms}$ at BOL to $\sim 11 \mathrm{ADU}_{15 \mathrm{bit}} \mathrm{rms} \approx 20 \mathrm{e}^{-} \mathrm{rms}$ at EOL.

\section{References}

Auvergne, M., Bodin, P., Boisnard, L., et al. 2009, A\&A, 506, 411

Baer, R. L. 2006, in Sensors, Cameras, and Systems for Scientific/Industrial Applications VII, ed. M. M. Blouke, Proc. SPIE, 6068, 606805

Baggett, S. M., Noeske, K., Anderson, J., MacKenty, J. W., \& Petro, L. 2012, in Proc. SPIE, 8453,

Bernardi, P., Lapeyrere, V., Buey, J.-T., et al. 2004, in Stellar Structure and Habitable Planet Finding, eds. F. Favata, S. Aigrain, \& A. Wilson, ESA SP, 538, 191

Bogaart, E. W., Hoekstra, W., Peters, I. M., Kleimann, A., \& Bosiers, J. T. 2009, IEEE Transactions on Electron Devices, 56, 2462

Box, G. E. P., \& Cox, D. R. 1964, J. Roy. Stat. Soc. Ser. B (Methodological), 26, 211 
Brown, T. M., \& Davies, J. E. 2003, in Proc. HST Calibration Workshop, Hubble after the Installation of the ACS and the NICMOS Cooling System, eds. S. Arribas, A. Koekemoer, \& B. Whitmore, 180

Buey, J.-T., Bernardi, P., Decaudin, M., et al. 2005, in Proc. SPIE, 5902, eds. T. J. Grycewicz, \& C. J. Marshall, 19

Burger, H., Scholköpf, B., \& Harmeling, S. 2011, in IEEE International Conference on Computational Photography (ICCP), 1

Burke, B., Jorden, P., \& Vu, P. 2005, Exp. Astron., 19, 69

Cai, Z., Wei, X., Ju, J., \& Wu, J. 2010, in Optoelectronic Imaging and Multimedia Technology, eds. Y. Toru et al., Proc. SPIE, 7850, 785005

Chugg, A., Jones, R., Moutrie, M., et al. 2003, IEEE Trans. Nucl. Sci., 50, 2011

Corbard, T., Boumier, P., Appourchaux, T., et al. 2008, Astron. Nachr., 329, 508

Corbard, T., Salabert, D., Boumier, P., et al. 2013, J. Phys. Conf. Ser., 440, 012025

Crisp, R. 2011, J. Electronic Imaging, 20, 033006

Defise, J.-M., Clette, F., Moses, J. D., \& Hochedez, J.-F. E. 1997, Proc. SPIE, 3114,598

Downing, M., Baade, D., Sinclaire, P., Deiries, S., \& Christen, F. 2006, in SPIE Conf. Ser., 6276

Dunlap, J. C., Bodegom, E., \& Widenhorn, R. 2011, in Sensors, Cameras, and Systems for Industrial, Scientific, and Consumer Applications XII, Proc. SPIE 7875, 78750

Dunlap, J., Blouke, M., Bodegom, E., \& Widenhorn, R. 2012, IEEE Transactions on Electron Devices, 59, 1114

Feynman, J., \& Gabriel, S. B. 2000, J. Geophys. Res., 105, 10543

Fryzlewicz, P. 2007, J. American Statistical Association, 102, 1318

Gilard, O., Boutillier, M., Quadri, G., Rolland, G., \& Germanicus, R. 2008, IEEE Trans. Nucl. Sci., 55, 3626

Gilard, O., Boatella-Polo, C., Dolado-Perez, J.-C., et al. 2010, IEEE Trans. Nucl. Sci., 57, 1644

Gomez Rodriguez, M., Kober, J., \& Schölkopf, B. 2009, in IEEE International Conference on Computational Photography

Gow, J. P. D., Murray, N. J., Holland, A. D., Burt, D., \& Pool, P. J. 2012, Nucl. Instrum. Methods Phys. Res. A, 686, 15

Hill, R. S., Landsman, W. B., Lindler, D., \& Shaw, R. 1997, in The 1997 HST Calibration Workshop with a New Generation of Instruments, eds. S. Casertano, et al., 120

Hopkins, I., \& Hopkinson, G. 1993, IEEE Trans. Nucl. Sci., 40, 1567

Hopkinson, G. R., Dale, C. J., \& Marshall, P. W. 1996, IEEE Trans. Nucl. Sci., 43,614

Hopkinson, G., Goiffon, V., \& Mohammadzadeh, A. 2007, in Radiation and Its Effects on Components and Systems, RADECS, 2007, 1

Ipatov, S., A’Hearn, M., \& Klaasen, K. 2007, Adv. Space Res., 40, 160
Janesick, J. R., Elliott, T., Collins, S., Blouke, M. M., \& Freeman, J. 1987a, Opt. Eng., 26, 692

Janesick, J. R., Klaasen, K. P., \& Elliott, T. 1987b, Opt. Eng., 26, 972

Kutner, M., Nachtsheim, C., \& Neter, J. 2004, Applied linear regression models, Irwin/McGraw-Hill series in operations and decision sciences (McGrawHill/Irwin)

Lapeyrere, V., Bernardi, P., Buey, J.-T., Auvergne, M., \& Tiphène, D. 2006, MNRAS, 365, 1171

Lo, D., \& Srour, J. 2003, IEEE Trans. Nucl. Sci., 50, 2018

Marshall, C. J., Marshall, P. W., Wacynski, A., et al. 2004, in SPIE Conf. Ser., 5499, eds. J. D. Garnett, \& J. W. Beletic, 542

Marshall, C., Marshall, P., Waczynski, A., et al. 2005, IEEE Trans. Nucl. Sci., 52, 2672

Massey, R., Stoughton, C., Leauthaud, A., et al. 2010, MNRAS, 401, 371

Meftah, M., Hochedez, J.-F., Irbah, A., et al. 2013, Sol. Phys.

Penquer, A., Boutillier, M., Rolland, G., et al. 2009, IEEE Trans. Nucl. Sci., 56, 2142

Polidan, E. J., Waczynski, A., Marshall, P. W., et al. 2004, in Proc. SPIE, 5167, eds. T. J. Grycewicz, \& C. R. McCreight, 258

Rest, A., Mundermann, L., Widenhorn, R., Bodegom, E., \& McGlinn, T. C. 2002, Rev. Scientific Instruments, 73, 2028

Rhodes, J., Leauthaud, A., Stoughton, C., et al. 2010, PASP, 122, 439

Rodricks, B. G., \& Venkataraman, K. 2005, in Proc. SPIE, 5678, eds. N. Sampat, J. M. DiCarlo, \& R. J. Motta, 59

Rousseeuw, P., \& Leroy, A. 2005, Robust Regression and Outlier Detection, Wiley Series in Probability and Statistics (Wiley)

Sakia, R. M. 1992, J. Roy. Stat. Soc. Ser. D (The Statistician), 41, 169

Schou, J. 2004, in Stellar Structure and Habitable Planet Finding, eds. F. Favata, S. Aigrain, \& A. Wilson, ESA SP, 538, 411

Sirianni, M., Mutchler, M., Clampin, M., et al. 2004, in SPIE Conf. Ser., 5499 eds. J. D. Garnett, \& J. W. Beletic, 173

Smith, G. E. 2010, Rev. Mod. Phys., 82, 2307

Srour, J. R., \& Palko, J. W. 2006, IEEE Trans. Nucl. Sci., 53, 3610

Srour, J., Marshall, C., \& Marshall, P. 2003, IEEE Trans. Nucl. Sci., 50, 653

Thuillier, G., Dewitte, S., \& Schmutz, W. 2006, Adv. Space Res., 1792, 38

Widenhorn, R., Blouke, M. M., Weber, A., Rest, A., \& Bodegom, E. 2002, in SPIE Conf. Ser. 4669, eds. M. M. Blouke, J. Canosa, \& N. Sampat, 193

Widenhorn, R., Hartwig, I., Dunlap, J. C., \& Bodegom, E. 2008, in SPIE Conf. Ser., 6816, 68160

Widenhorn, R., Dunlap, J., \& Bodegom, E. 2010, IIEEE Trans. Nucl. Sci., 57, 581

Widenhorn, R., Rest, A., Blouke, M. M., Berry, R. L., \& Bodegom, E. 2007, in Proc. SPIE, 6501, 650103 